\title{
Ultrawideband Radio Design: The Promise of High-Speed, Short-Range Wireless Connectivity
}

\author{
SUMIT ROY, JEFF R. FOERSTER, V. SRINIVASA SOMAYAZULU, AND DAVE G. LEEPER
}

Invited Paper

This paper provides a tutorial overview of ultrawideband (UWB) radio technology for high-speed wireless connectivity. Subsequent to establishing a historical and technological context, it describes the new impetus for UWB systems development and standardization resulting from the FCC's recent decision to permit unlicensed operation in the $[3.1,10.6] \mathrm{GHz}$ band subject to modified Part 15 rules and indicates the potential new applications that may result. Thereafter, the paper provides a system architect's perspectives on the various issues and challenges involved in the design of link layer subsystems. Specifically, we outline current developments in UWB system design concepts that are oriented to high-speed applications and describe some of the design tradeoffs involved.

Keywords-Personal area networks, wireless, ultrawide band.

\section{INTRODUCTION AND BACKGROUND}

Ultrawideband (UWB) technology is at present defined by the Federal Communications Commission (FCC) as any wireless transmission scheme that occupies a fractional bandwidth $W / f_{c} \geq 20 \%$ where $W$ is the transmission bandwidth and $f_{c}$ is the band center frequency, or more than $500 \mathrm{MHz}$ of absolute bandwidth. In traditional UWB systems, such large bandwidths were achieved by using very narrow time-duration baseband pulses of appropriate shape and duration - these include the family of Gaussian shaped pulses and their derivatives [2]. To confine the transmitted spectrum to a desired passband, postfiltering of the baseband pulse spectrum including the impact of the transmit antenna characteristics has traditionally been assumed. There are several benefits to using such large bandwidths-in an additive Gaussian noise-limited environment, expanding

Manuscript received August 6, 2003; revised November 10, 2003. This work was completed while S. Roy was on academic leave at Intel Wireless Technology Lab, Hillsboro, OR 97124-5961 USA.

S. Roy is with the University of Washington, Seattle, WA 98101 USA. (e-mail: roy@ee.washington.edu).

J. R. Foerster, V. S. Somayazulu, and D. G. Leeper are with the Wireless Technology Division, Intel Labs, Hillsboro, OR 97124-5961 USA.

Digital Object Identifier 10.1109/JPROC.2003.821910

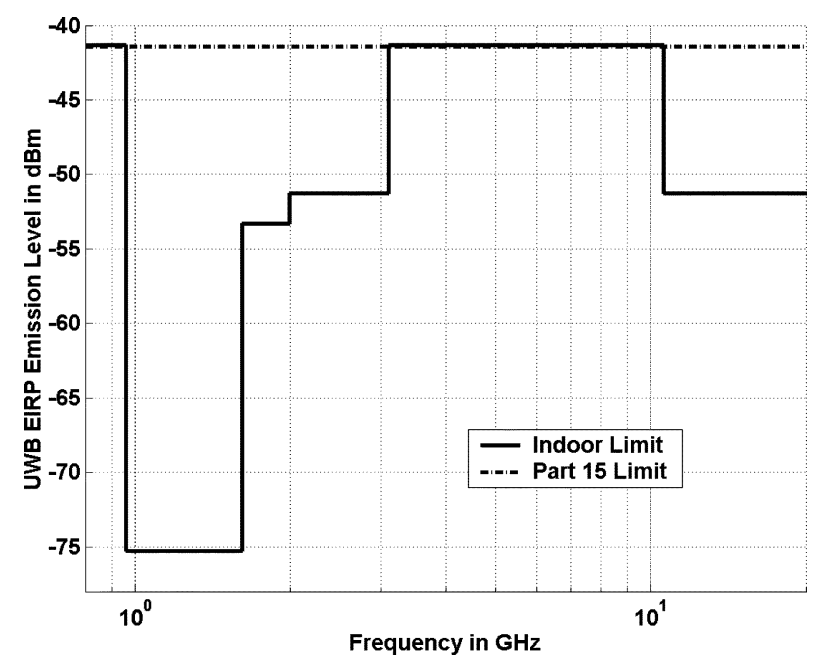

Fig. 1. UWB spectral mask and FCC Part 15 limits.

bandwidth (when feasible) is the preferred cost-effective pathway to achieving higher data rates without the need to increase transmit power (or equivalently, resorting to sophisticated error control coding and higher order modulation schemes). In a multipath dominated environment, larger transmission bandwidths result in the ability for increasingly fine resolution of multipath arrivals, which leads to reduced fading per resolved path, since the impulsive nature of the transmitted waveforms prevents significant overlap and, hence, reduces possibility of destructive combining.

The FCC recently approved [1] the deployment of UWB ${ }^{1}$ on an unlicensed basis in the $3.1-10.6 \mathrm{GHz}$ band subject to a modified version of Part 15.209 rules. The essence of this ruling is to limit the power spectral density (PSD) measured in a $1-\mathrm{MHz}$ bandwidth at the output of an isotropic transmit antenna to that shown in Fig. 1. The above spectral mask allows UWB-enabled devices to overlay existing

\footnotetext{
${ }^{1}$ At the time of writing, UWB is being considered in both Europe and Japan for unlicensed bands but is yet to be approved.
} 


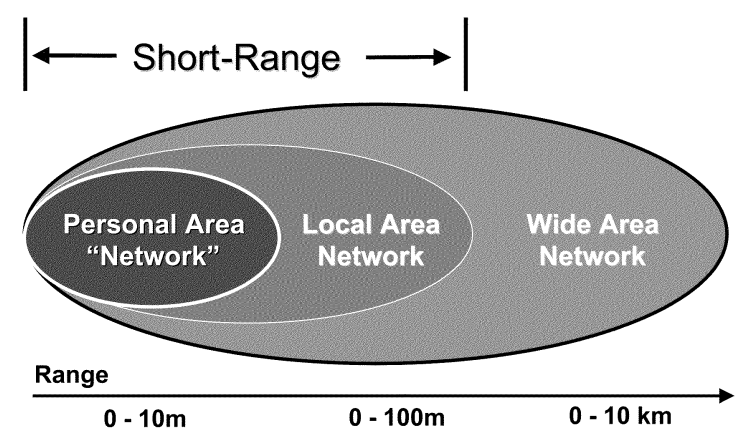

Fig. 2. WPAN, WLAN, and cellular networks: typical link ranges.

systems while ensuring sufficient attenuation to limit adjacent channel interference. ${ }^{2}$ Additional PSD limits have been placed below $2 \mathrm{GHz}$ to protect critical applications such as global positioning system (GPS) as shown. ${ }^{3}$ The first consequence of this spectral mask imposed by the FCC is to render the use of baseband pulse shapes difficult without additional transmit filtering to limit the out-of-band emission spectra.

In summary, UWB communications is allowed at a very low average transmit power ${ }^{4}$ compared to more conventional (narrowband) systems that effectively restricts UWB to short ranges. UWB is, thus, a candidate physical layer mechanism for IEEE 802.15 Wireless Personal Area Network (PAN) for short-range high-rate connectivity that complements other wireless technologies in terms of link ranges, as shown in Fig. 2.

\section{A. Dynamic Spectrum Utilization}

The allocation of such a large chunk of spectrum for unlicensed use signals a significant shift from a regulatory viewpoint that has hitherto been dominated by licensed spectrum usage philosophy-initially awarded free guided by prevailing notions of "public airwaves" (broadcast television in VHF/UHF bands) or later via an auctioning mechanism (voice telephony in cellular and PCS bands). This action has been significant enough to raise many concerns ${ }^{5}$ on several fronts-notably regarding UWB's ability to coexist with legacy services such as IEEE 802.11a wireless local area networks (WLANs), radar systems, etc., that it will overlay as well as sensitive military and civilian services in adjacent bands such as GPSs and other aircraft navigation systems.

However, a broader perspective (one that weighs acceptable impacts on existing systems with the greater common net good obtained by introducing such new overlay-friendly technologies such as UWB) based on total spectrum utiliza-

\footnotetext{
${ }^{2}$ Notably, the PCS bands for digital cellular at $1.9 \mathrm{GHz}$ and GPS band at $1.5 \mathrm{GHz}$.

${ }^{3}$ It should be noted that the current FCC ruling is open to possible future modification based on comments and review; these may include, for example, increasing the upper band edge as well as additional constraints as deemed necessary to protect legacy services in adjacent bands.

${ }^{4} \mathrm{At}-41 \mathrm{dBm} / \mathrm{MHz}$ constant PSD over a $7.5-\mathrm{GHz}$ bandwidth implies approximately $0.55 \mathrm{~mW}$ average transmit power.

${ }^{5}$ During the commentary period of the Notice of Proposed Rule Making (NPRM), the FCC received a large number of responses to the docket, many from concerned licensed service providers, indicating the intense interest that UWB has generated.
}

tion viewpoint reveals the potential for achieving more efficient spectrum utilization. ${ }^{6}$ The market success of 802.11 WLAN technology has led to calls for increasing globally harmonized allocations of unlicensed spectrum. It is likely that addition of such new spectrum for unlicensed use will require an etiquette for sharing of common resources via mechanisms such as dynamic spectrum management facilitated by ongoing advances in agile software-defined radio architectures that can adapt radio hardware and associated firmware to configure transceivers best suited to existing conditions. Hence, the ability of UWB to fill-in unused/underused spectrum at any time promotes opportunistic communications can contribute to both greater spectral efficiency and aggregate network throughput assuming a suitable multiple accessing network architecture for UWB is identified.

\section{B. Historical Context and Applications}

Modern UWB technology has been around since the 1960s but with a very different focus than the thrust of this paper-early UWB development centered on impulse radar (see [2] and [3] and references therein) developed largely under the aegis of the U.S. Department of Defense that used wideband signals primarily for very accurate localization and imaging in the context of secure communications. This has given rise to a thriving commercial and military sector of UWB components and products that exploit the fine locationing capabilities of UWB. ${ }^{7}$ Within the academic context, UWB investigations were largely pioneered by Prof. Scholtz and his group [5], [7], [8] since the early 1990s, focusing mainly on low-rate applications.

Recent developments in high-speed switching and narrowband pulse generation technology has prompted a fresh look at UWB signal generation for very different purposes: high-speed, short-range networking in support of a variety of potential low-cost, low-power multimedia transport applications in home and enterprise environments. One typical scenario is provisioning wireless data connectivity between a host (e.g., desktop PC) and associated peripherals (keyboard, mouse, printer, etc.); in this mode, a UWB link essentially functions as "cable replacement" with transfer rate requirements that range from a relatively low (order of $100 \mathrm{~Kb} / \mathrm{s}$ for a wireless mouse) to high $(100 \mathrm{Mb} / \mathrm{s}$ for rapid file sharing or download of images/graphic files where reduced latency will be the overriding concern). Additional driver applications relates to streaming of digital media content between consumer electronics appliances (digital TV sets, VCRs, audio CD/DVD and MP3 players, etc.) that require significant data rates (on the order of $10 \mathrm{Mb} / \mathrm{s}$ per flow) with quality-of-service (QoS) guarantees in terms of delay and delay jitter. UWB may also support direct peer-to-peer communications between mobile wireless devices such as laptops and PDAs that occasionally exchange

\footnotetext{
${ }^{6}$ Studies of licensed bands have shown that a significant percentage of spectrum remains unused, averaged over time, contributing to this spectral inefficiency.

${ }^{7}$ See, for example, see http://www.timedomain.com and http://www.aetherwire.com regarding technology and products from TimeDomain Corp. and AetherWire and Locationing Inc., respectively.
} 
information in an $a d$ hoc, as-needed manner. This has naturally led to investigations into feasible network architectures for an extended network of such mobile, power constrained devices that provide end-to-end connectivity in a multihop manner. The varied nature of the above applications places a premium on scalability and adaptability of any system architecture as an important criterion for standards-based adoption. In summary, UWB is seen as having potential for realizing an exciting new set of applications that to date have not been fulfilled by other wireless short range technologies currently available (e.g., 802.11 LANs and Bluetooth PANs); this has lead to the formation of the IEEE 802.15.3a Task Group, tasked with developing a UWB-based physical layer standard for component (radio) development that conforms to the 15.3 MAC. This has resulted in significant industry interest as indicated by the formation of various new privately funded startup organizations as well as interest from established silicon vendors all aiming to develop new UWB chipsets. Correspondingly, there has been a recent upsurge in academic research into UWB as evidenced by [33] and an annual conference [34] solely dedicated to UWB technology.

As can be expected, numerous approaches toward exploiting the available bandwidth to support practical designs have been proposed in the literature-it is not our intent (and neither is it feasible) in this review to exhaustively enumerate all; rather, we seek to highlight the key principles and motivations behind the principal approaches from a systems design perspective and illustrate the tradeoffs that ensue when UWB is applied to the goal of high data rate wireless connectivity.

The rest of the paper is organized as follows. Section II provides a high-level context to that relates UWB to other short-range wireless technologies, while Section III focusses on capacity and link budget considerations for UWB. Detailed design issues of important transceiver subsystems are contained in Section IV including an up-to-date description of UWB channel modeling. Section V concludes the paper with a description of future system design challenges.

\section{Figures OF MERIT FOR WIRELESS NETWORKS}

Wireless network design involves well-known tradeoffs between: 1) desired coverage (range); 2) achievable data rates (b/s); and 3) system figures of merit (cost, complexity, and power budget). For the communication link design, metrics such as bandwidth efficiency measured in bits/s/Hz and power efficiency measured in required $E_{b} / N_{0}$ to achieve the desired rate/range operating point are traditionally used. A useful figure of merit for networks is the spatial capacity $\left(\mathrm{b} / \mathrm{s} / \mathrm{m}^{2}\right)$ that captures the notion of aggregate data intensity in time and space, and serves as a means of comparing different short-range wireless technologies.

An intuitive understanding of the tradeoffs is obtained by comparing short-range technologies against wide area networks such as cellular telephony that cover distances of the order of several kilometers with high power and costly system components. Connectivity of shorter distances such as in WLANs (ranges up to $100 \mathrm{~m}$ ) and WPANs (ranges up to $10 \mathrm{~m}$ ) potentially allow for greater spectrum reuse and thereby support more users in a given area provided the chosen network architecture and multiple accessing scheme can effectively manage the enhanced interference. Driven by the high-speed, short-range data networking applications in a home environment, WPANs are, thus, of great interest as a scalable networking solution while maintaining reasonable cost and reduced power consumption by exploiting advances in digital signal processing (DSP) hardware and Moore's Law for silicon integration.

\section{Example}

The 2.4-GHz industrial, scientific, and medical (ISM) band contains $80 \mathrm{MHz}$ of usable spectrum, implying that three 22-MHz IEEE 802.11b systems can operate on a noninterfering basis, each offering a peak rate of $11 \mathrm{Mb} / \mathrm{s}$ for a total aggregate speed of $33 \mathrm{Mb} / \mathrm{s}$. For a operating range of $100 \mathrm{~m}$, this yields a spatial capacity of approximately 1 $\mathrm{Kb} / \mathrm{s} / \mathrm{m}^{2}$. Bluetooth in its low-power mode has a rated $10-\mathrm{m}$ range and a peak over-the-air speed of $1 \mathrm{Mb} / \mathrm{s}$. Studies have shown that approximately ten Bluetooth clusters can operate simultaneously in this range with minimal degradation yielding an aggregate speed of $10 \mathrm{Mb} / \mathrm{s}$ [23] for a spatial capacity of approximately $30 \mathrm{~Kb} / \mathrm{s} / \mathrm{m}^{2}$. UWB systems are being currently designed for $110 \mathrm{Mb} / \mathrm{s}$ at $10-\mathrm{m}$ range with four collocated clusters - this projects to spatial capacity of about $1.3 \mathrm{Mb} / \mathrm{s} / \mathrm{m}^{2}$. Thus, UWB potentially supports data intensity that are several orders of magnitudes larger than present-day WLANs/WPANs, although in terms of spectral efficiency $(\mathrm{b} / \mathrm{s} / \mathrm{Hz})$ alone, UWB is significantly poorer. Thus, UWB represents a tradeoff between lower spectral efficiency for increased power efficiency to achieve a given rate/range operating point with limited transmit power.

\section{Single User In AdDitive White Gaussian Noise: CAPACITY AND LinK Budget CONSIDERATIONS}

\section{A. Capacity}

In traditional narrowband communications (where the fractional bandwidth is of the order of $1 \%$ or less), the received PSD can be related to the transmit PSD by the Friis formula that accounts for two loss components: 1) a distance dependance loss component governed by the average path loss exponent and 2) a frequency dependant loss component that is assumed constant over the band. Due to the very large UWB signal bandwidth, the second component can no longer be strictly approximated as a constant over the entire bandwidth. Thus, the received signal-to-noise ratio (SNR) PSD in additive white Gaussian noise (AWGN) $\operatorname{SNR}_{r}(f)$ is given by

$$
\begin{aligned}
& \operatorname{SNR}_{r}(f)(\mathrm{dB}) \\
& =-41-(-108)-L-20 \log _{10}\left(\frac{4 \pi f}{c}\right)-\gamma 10 \log _{10} d
\end{aligned}
$$

where $c=3 \times 10^{8} \mathrm{~m} / \mathrm{s}$ is the speed of light, $\gamma$ is the exponent of path loss, $d$ the distance to the receiver from the 


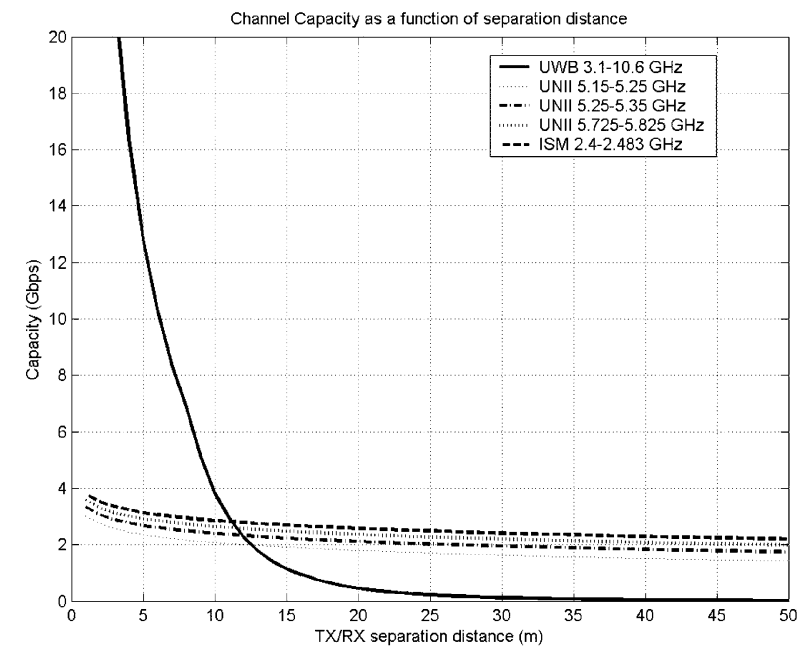

Fig. 3. UWB capacity versus other WLAN technologies.

source, $L(\mathrm{~dB})$ subsumes all additional system implementation losses, and $-41 \mathrm{dBm} / \mathrm{MHz}$ is the allowed emitted PSD per Part 15 rules. A 6-dB noise figure was applied to the $N_{0}=-114 \mathrm{dBm} / \mathrm{MHz}$ standard thermal noise PSD in the above computation as representing a more realistic assessment of the SNR at the receiver input.

By Shannon's formula for the capacity of a single user in additive white Gaussian noise (in bits per second)

$$
C(d)=B \log _{2}\left(1+\langle\mathrm{SNR}\rangle_{r}(d)\right)
$$

where $B=10.6-3.1=7.5 \mathrm{GHz}$ and an average (over frequency) received $\langle\mathrm{SNR}\rangle_{r}$ is obtained from (1) using the center frequency $f_{c}=3.1+10.6 / 2=6.85 \mathrm{GHz}$. The results of computation of (2) are shown in Fig. 3 where the path loss exponent is assumed equal to 2 (free space) up to 8 $\mathrm{m}$, and 3.3 beyond, as representative of some indoor wireless channels.

For comparison purposes, achievable rates for two other short-range wireless networking technologies-notably 802.11a in the UNII $(5-\mathrm{GHz})$ band and Bluetooth in the 2.4-GHz ISM bands-are also shown. The first insight provided by the above is that UWB provides the highest potential link layer rates vis-à-vis other wireless technologies at shorter distances (typically $<10 \mathrm{~m}$ ) beyond which a crossover occurs. The low transmit PSD naturally limits UWB to short ranges; however a particularly important degree of freedom in UWB channelization is the variable bandwidth while maintaining a constant PSD. In contrast, the FCC limits the maximum average transmit power for the UNII and $2.4 \mathrm{GHz}$ bands: 1) 16, 23, $29 \mathrm{dBm}$, respectively, for three UNII Bands: (5.15-5.25) GHz, (5.25-5.35) GHz, and (5.725-5.825) GHz and 2) $30 \mathrm{dBm}$ for 2.4-GHz ISM Band (2.4-2.483 GHz). This implies that the PSD is decreased as the channel bandwidth increases in these cases in contrast to UWB, thereby allowing UWB-based link layers to potentially achieve very high (gigabit) rates.

The conclusion is that UWB is better suited for throughput optimization at 1-10-m ranges, and conversely, other
(WLAN and non-UWB WPAN) technologies are more appropriate for longer distances. Finally, the results provide reasons for cautious optimism in anticipating gigabit connectivity at short ranges with UWB. It is important not to overstate the utility of single-user capacity in AWGN analysis - this is only a starting point for practical systems design and all practical applications of UWB will occur in dense multipath environments with many simultaneous UWB and non-UWB users. Thus, estimates of achievable aggregate bit rates as a function of bandwidth scaling for various multiple access strategies call for continuing information theoretic investigations on the lines of the recent work of [17]-[19].

\section{B. Link Budget Analysis}

While capacity calculations quantify an ideal distance versus throughput relationship based on the assumptions used, achievable rates based on a pragmatic link budget analysis is a better indicator of what is feasible with state-of-the-art designs. We consider the pulse amplitude modulated (PAM) UWB system to highlight some of the tradeoffs with UWB based on the following assumptions.

1) AWGN channel with a target bit error rate (BER) of $10^{-3}$ for uncoded transmission is used. ${ }^{8}$

2) Transmit PSD is limited to $-41 \mathrm{dBm} / \mathrm{MHz}$ (per Part 15.209).

3) An antenna gain of $0 \mathrm{dBi}$ is assumed.

4) A 5-dB link margin is assumed.

5) A 6-dB noise figure is assumed along with a 3-dB implementation loss.

6) Operating bandwidth is approximately $3 \mathrm{GHz}$ (from 3.1-6 GHz to allow operation in the $2.4 \mathrm{GHz}$ ISM band and the $5 \mathrm{GHz}$ U-NII band); the band center frequency is used for computing the distance loss function.

7) Free space propagation (i.e., path loss is proportional to the square of the propagation distance) is used primarily for illustrative purposes only, ${ }^{9}$ which results in a path loss given by $L(d)=20 \log (4 \pi / \lambda)+20 \log (d)$ where $\lambda$ is the carrier wavelength corresponding to the center frequency.

The probability of symbol error for an uncoded M-PAM system is given by (assuming coherent detection) [32]

$$
P_{e, M} \approx \operatorname{erfc}\left(\sqrt{\frac{3 k \gamma_{b}}{M^{2}-1}}\right)
$$

where $M=2^{k}$ and $\gamma_{b}$ is the SNR per bit (the SNR per symbol is $\left.E_{s} / N_{0}=k \gamma_{b}\right), N_{0}$ is the usual thermal noise PSD and erf(.) is the familiar complementary error function. The corresponding probability of bit error is estimated by $P_{b}=(1 / k) P_{e, M}$.

To get a better understanding of the tradeoffs by varying the pulse bandwidth and pulse repetition period,

\footnotetext{
${ }^{8}$ With suitable coding, this should reduce to $10^{-6}$ or better at the cost of reducing the overall data throughput.

${ }^{9}$ For indoor channels at these frequency bands, an exponent around 3.0 is more appropriate.
} 


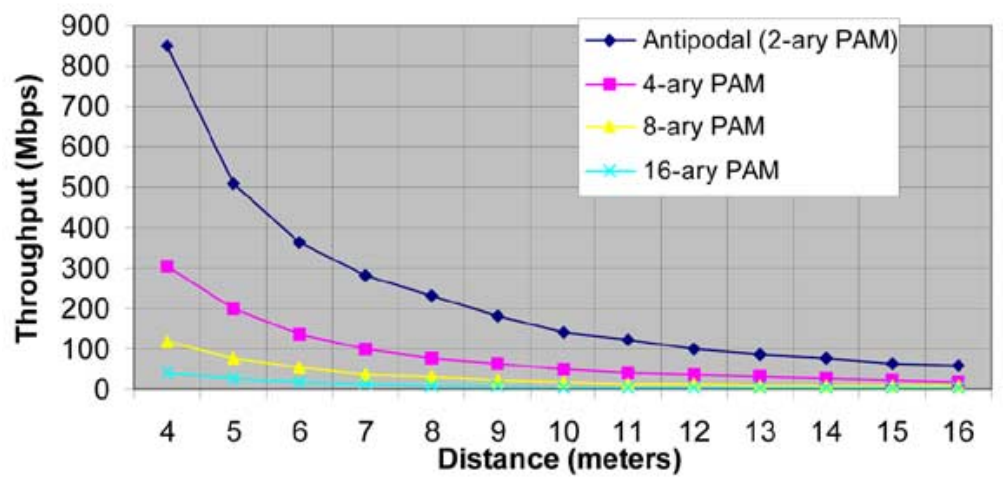

Fig. 4. Link budget comparisons for M-PAM modulation schemes.

Table 1

Required $E_{b} / N_{0}$ for PAM Modulation

\begin{tabular}{c|c|c|c}
\hline $\mathrm{k}$ & $\mathrm{M}$ & $\gamma_{b}(\mathrm{~dB})$ & $\frac{E_{s}}{N_{0}}(\mathrm{~dB})$ \\
\hline 1 & 2 & 7 & 7 \\
\hline 2 & 4 & 10.75 & 13.75 \\
\hline 3 & 8 & 15 & 19.77 \\
\hline 4 & 16 & 19.5 & 25.5 \\
\hline
\end{tabular}

rewrite the SNR per symbol as $E_{s} / N_{0}=P_{\mathrm{av}} T_{r} / N_{0}=$ $\left(P_{\mathrm{sd}} / N_{0}\right)\left(B_{s} / B_{r}\right)$ where $P_{\mathrm{av}}=B_{s} P_{\mathrm{sd}}$ is the average transmitted power in a symbol interval, $P_{\text {sd }}$ is the PSD limit as set by Part $15, B_{s} \approx 1 / T_{d}$ is the signal bandwidth and $B_{r}=1 / T_{r}$ is the pulse repetition frequency (PRF). Note that increasing the processing gain $\mathrm{PG}=B_{s} / B_{r}$ either via increasing the system bandwidth $B_{s}$ or reducing the PRF $B_{r}$ increases the available link margin for a given range (or equivalently, increases the given range for a fixed transmit PSD). This has the effect of increasing the peak transmit power while allowing UWB to operate at a very low average transmit PSD.

From well-known analysis [32], we have Table 1, which is a table of required $E_{s} / N_{0}$ for a target uncoded $P_{b}=10^{-3}$.

Note that if the pulse bandwidth is kept fixed (equivalently, the average transmit power is constant) as in the present calculation, the pulse repetition period between the symbols will need to increase, thereby reducing the data rate. The above yields the throughput versus distance graph in Fig. 4 that is a better design guide than the capacity curve for particular choice of modulation schemes. The ability of UWB to provide simple and effective mechanism for rate/range adaptation is a key implementation advantage-for example, as the range increases, a UWB radio may use several pulses to send one information bit, thereby increasing the SNR at the receiver. Since the average power consumption of a UWB transmitter grows linearly with PRF, it is feasible to envision a relatively simple UWB radio that, under software control, can dynamically trade data rate, power consumption, and range.

The results here suggest that higher order M-PAM systems do not improve the throughput as much as using lower order 2-PAM with a higher PRF. This can be understood by re- calling that PAM is a very spectral efficient modulation technique, but not very power efficient. For UWB systems, the spectrum is determined more by the shape of the pulse rather than the symbol rate. Therefore, for an AWGN channel, it is reasonable to expect that lower order PAM would result in the best performance. However, considerations for a multipath channel can differ due the fact that the 2-PAM system would experience greater intersymbol interference as compared to higher order systems with greater pulse repetition periods, that would potentially limit throughput.

\section{Design GoAls AND CONSIDERATIONS FOR UWB PHYSICAL LAYER}

In this section, we first provide a summary of the link layer goals for UWB-based WPAN networks.

\section{A. 15.3 UWB Physical Layer and MAC Architecture Overview}

The 15.3 physical layer criterion [14] requires designs to achieve $110 \mathrm{Mb} / \mathrm{s}$ for a receiver at distance $d=10 \mathrm{~m}$ and 200 $\mathrm{Mb} / \mathrm{s}$ at $d=4 \mathrm{~m}$, with options for demonstrating scalability to higher speeds of up to $480 \mathrm{Mb} / \mathrm{s}$ at distances $<4 \mathrm{~m}$. These speeds are to be demonstrated for the four SG3a channel models specified in [13] that cover a variety of line-of-sight (LOS) and non-line-of-sight (NLOS) scenarios for a packet error rate (PER) of (no greater than) $8 \%$ for 1-KB packets. Since coexistence with various in-band non-UWB interferers is key priority for UWB, the above PER must be maintained for a reference interferer located $1 \mathrm{~m}$ away from the intended UWB receiver. Further, WPAN architectures composed of multiple simultaneous uncoordinated piconet operation are anticipated (corresponding to collocated clusters of different UWB devices that form their own network) implying the need for the design to be robust to multiple-access interference (MAI) from other UWB transmitters. Each piconet may comprise up to 256 client devices that are associated via a piconet coordinator (PNC). Once devices associate to form a piconet, the PNC is invested with the important task of maintaining piconet operation including transmitting beacons that carry important piconet parameter information that allows new devices to join as well as allocate resources for channel 

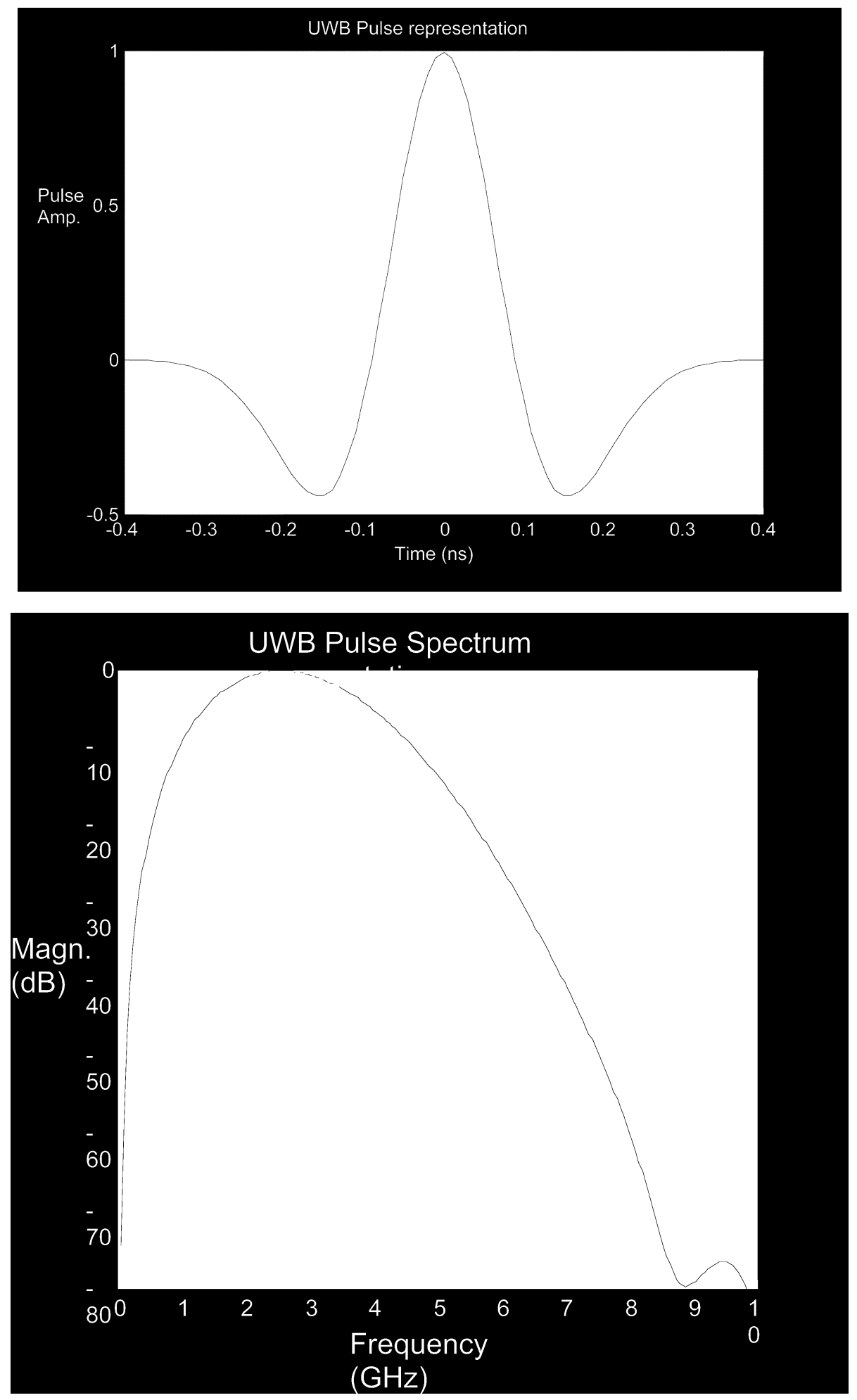

Fig. 5. UWB pulse waveform: Gaussian monocycle in time and frequency domain.

access to existing devices in the piconet. The 15.3 MAC adheres to a TDMA superframe format (typical duration of a few milliseconds) with two distinct components: 1) a contention access period (CAP) that allows for random access using carrier sense multiple access with collision avoidance (CSMA/CA) for nondelay critical applications such as asynchronous data and 2) a contention-free period (CFP) containing guaranteed time slots (GTS) based on client reserva- tion requests for support of real-time traffic flows. The relative duration of CAP and CFP can be dynamically adjusted on a per-frame basis as required by traffic demands.

We next describe the main subsystems in a UWB transceiver design: 1) pulse shaping and modulation;2) multiple accessing; 3) multipath channel characterization; 4) performance in multipath; 5) coexistence with narrowband sources; and 6) timing synchronization. The intent is not to provide an 

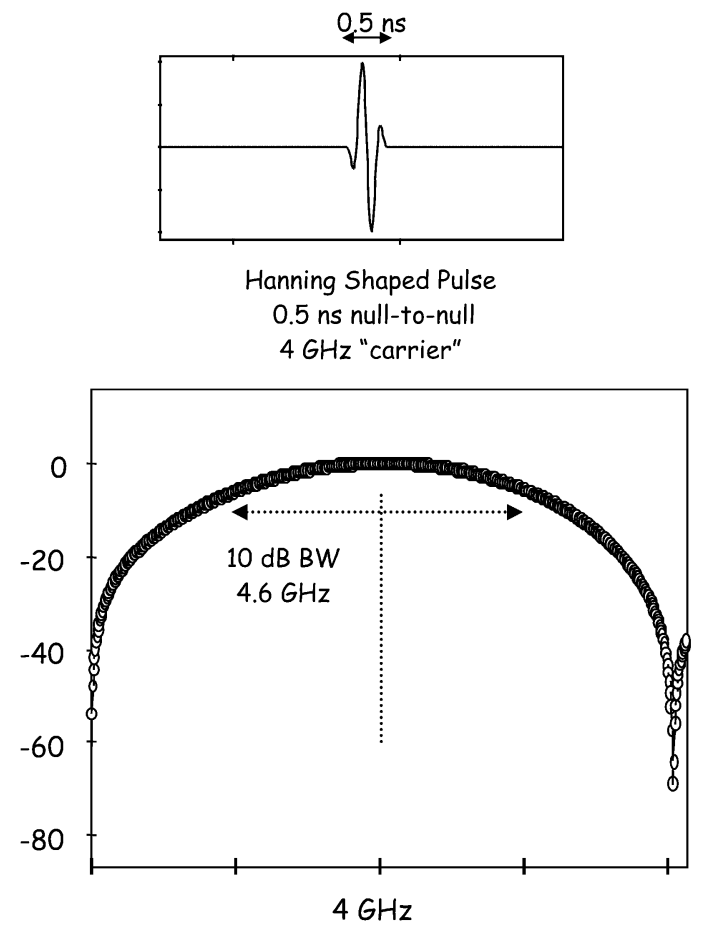

Normalized Power

Spectral Density

Fig. 6. UWB pulse waveform: Hanning windowed RF carrier.

exhaustive overview of all possible approaches but rather to provide insights into the main system tradeoffs by considering canonical cases.

\section{B. Pulse Shaping and Modulation}

UWB implementations directly modulate an impulse-like waveform with sharp rise/fall times that occupies several $\mathrm{GHz}$ of bandwidth. In earlier work, a typical baseband UWB pulse (see Fig. 5) such as the Gaussian monocycle obtained by differentiation of the standard Gaussian waveform has been used frequently for analytical evaluation of UWB systems. The waveform is given by

$$
u_{g}(t)=A\left[1-4 \pi\left(\frac{t}{T_{d}}\right)^{2}\right] e^{-2 \pi\left(t / T_{d}\right)^{2}}
$$

where the amplitude $A$ can be used to normalize the pulse energy (note the odd symmetry of the pulse shape that ensures a zero dc level). Note that the pulse Fourier transform possesses significant energy from near dc up to the system bandwidth $B_{s} \approx 1 / T_{d}$, as shown in Fig. $5 .{ }^{10}$ Clearly, such baseband pulses are unsuited to the FCC spectral mask, and point to the need for alternate pulse shapes. One such wideband pulse that is better suited to spectral control within the mask is shown in Fig. 6-it is characterized by two parameters $f_{c}, f_{s}$ where $f_{c}$ is the desired center-of-band frequency and $f_{s}$ is a modulation frequency that primarily impacts the

\footnotetext{
${ }^{10}$ For such baseband pulses, UWB system bandwidth is largely determined by the effective pulse duration and, to a lesser extent, by the pulse shape.
}

bandwidth of the transmitted signal. The pulse is generated by modulating an RF carrier at the desired center frequency $f_{c}$ with a Hanning window, i.e.,

$$
u_{h}(t)=0.5\left[1-\cos \left(2 \pi f_{s} t\right)\right] \cos \left(2 \pi f_{c} t\right)
$$

The main upshot of the link budget analysis in the previous section based on the Part 15 transmit PSD limitations and typical path loss models is that lower order modulations are feasible in practice at desired rate-distance operating points. Thus, UWB is primarily power constrained (and bandwidth unconstrained) implying that power efficiency is the critical constraint behind choice of modulation/coding approaches. Hence this work essentially limits itself to binary modulation-for obvious reasons of power efficiency, antipodal pulse signalling is preferred over on-off keying. When higher order modulation is needed to support increased data rates, $M$-ary orthogonal codes would be likely candidates due to their power efficiency.

Other than PAM, binary impulse modulation can also be achieved via altering the position of the pulse within a repetition period of $T_{r} \mathrm{~s}$ in response to information bits as shown in Fig. 7. Such pulse position modulation (PPM) in conjunction with time hopping was proposed by [5] and is discussed further in the subsequent section. A potential problem with binary PPM as evidenced by the transmit PSD shown in Fig. 7 is the presence of spectral lines or spikes which could violate the Part 15 constraints and must be suppressed by additional measures.

\section{Multiple Accessing}

Impulse modulation leads to "time spreading" between pulses that naturally allows for time multiplexing of users (equivalently, this can be thought of as orthogonal PPM or interleaved TDM and is a random access mechanism) provided the user impulses at the receiver do not overlap. The "spreading" or processing gain $P G=T_{r} / T_{d}$, thus, impacts the number of users that can be accommodated—reducing $T_{r}$ to increase the data rate per user reduces the number of users that can be supported, indicating a fundamental tradeoff.

Two approaches to UWB multiple accessing that have been discussed in the literature [4], [5] are based on: 1) traditional direct sequence (DS) encoding [4], [27] and 2) time-hopped PPM (TH-PPM) [5] that are shown in Fig. 7. In DS-UWB modulation, $N_{s}$ pulses or "chips" are sent per bit in a $T_{r}$ duration. The chip pulse sequence corresponds to a short (i.e., periodically repeated in each bit interval) binary pseudo-random code sequence $\mathbf{c}^{k}$ for the $k$ th user, analogous to familiar code division multiple access (CDMA). Note that the chip duration $T_{c}=T_{r} / N_{s}$ and that the data rate for binary modulation is $1 / T_{r}$.

In contrast, TH-PPM modulation uses the $k$ th user's code sequence $\mathbf{c}^{k}$ to randomly shift the position of the pulse during each repetition period in units of chip interval $T_{c}$ as shown; each code element is an integer in the range $0 \leq c_{j}^{k}<N_{h}$ where $N_{h}$ is naturally chosen to satisfy $N_{h} T_{c} \leq T_{r}$. The data rate in TH-PPM is $1 / N_{s} T_{r}$, since a bit duration now 

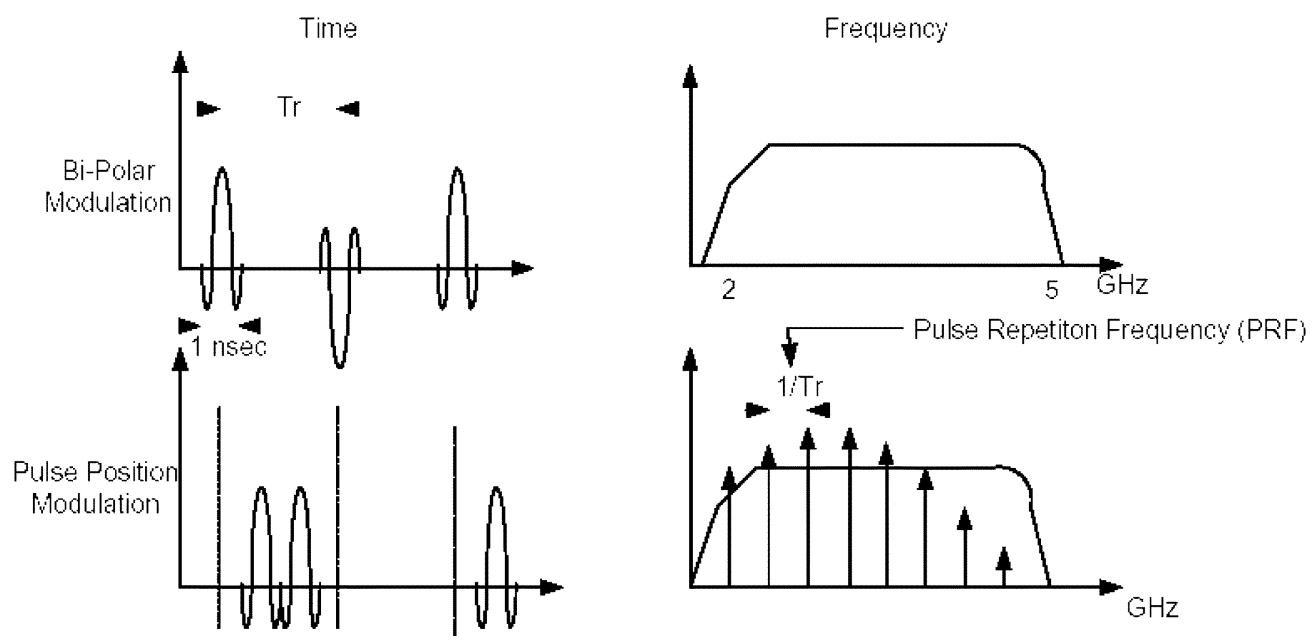

Fig. 7. Multiple access in UWB: DS and TH-PPM.

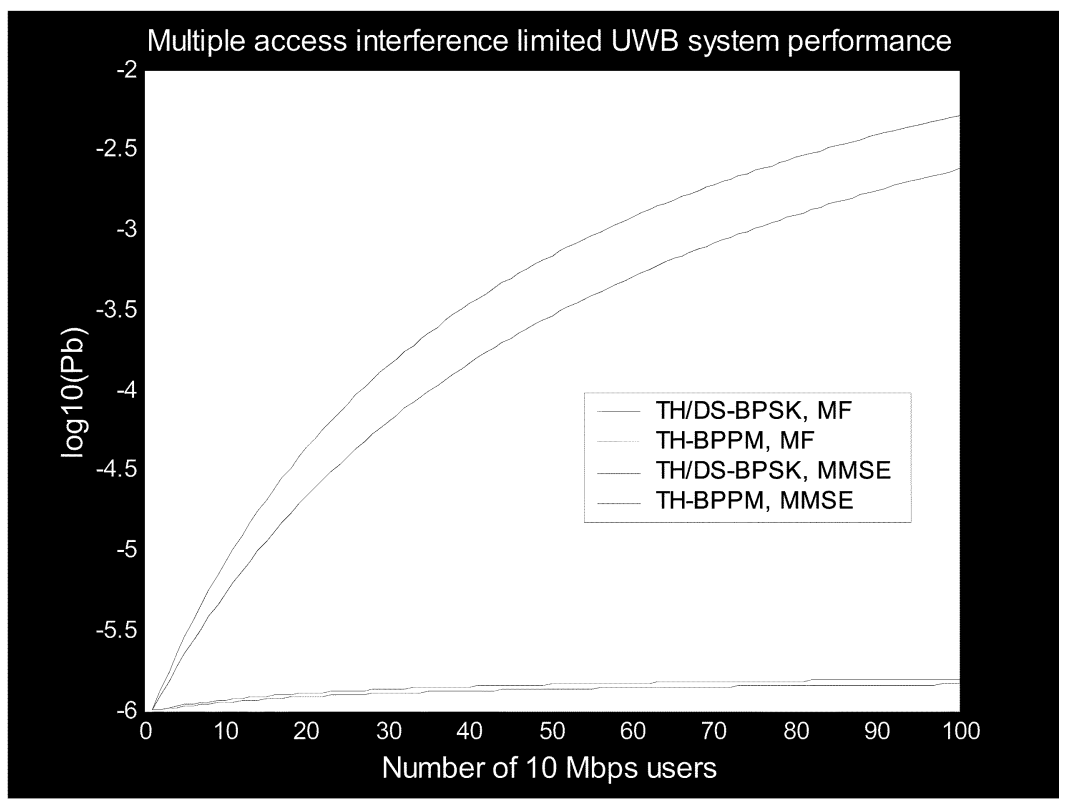

Fig. 8. Multiple access capacity of DS-UWB and TH-PPM.

spans $N_{s}$ intervals and the transmitted signal for the $k$ th user is given by

$$
s^{k}(t)=\sqrt{E_{k}} \sum_{i} u\left(t-i T_{r}-c_{j}^{(k)}-\delta d_{\left[j / N_{s}\right]}^{(k)}\right.
$$

where [.] denotes the integer part of the argument. The additional time offset $\delta d_{\left[j / N_{s}\right]}^{(k)}$ to the pulse position corresponds to modulation of the data with a step size of $\delta$.

The multiple accessing capability of these approaches depends, as can be expected, on a variety of factors that contribute to MAI at the decoder input, namely, the properties of the respective sequence design and the type of receiver used. The pros and cons of these two approaches is captured in Fig. 8 [26], which shows the BER degradation as function of the MAI for two types of receivers - an optimal single user receiver such as the matched filter and a suitable multiuser detector such as the minimum mean-squared error (MMSE) detector. The main conclusions can be summarized as follows.
1) With a matched filter receiver, DS-UWB multiple access is more suited for higher rates, as it can accommodate more users compared to TH-PPM for a given BER. At lower data rates, the multiple accessing capacity of the two systems are approximately the same; in such cases, TH-PPM may be preferred to DS-UWB, since it is potentially less susceptible to the near-far effect.

2) For a multiuser detector, the system capacity of the two approaches are approximately the same. In this context, it is worthwhile noting that the MAI for TH-PPM appears to be more non-Gaussian than a comparable DS-USB system ${ }^{11}$ with typical code sequences; hence, a combination of improved orthogonal TH-PPM codes as well as multiuser detection can be expected to provide greater gains.

${ }^{11}$ See, for example, [16], which investigates the Gaussianity assumption of MAI in TH-PPM. 


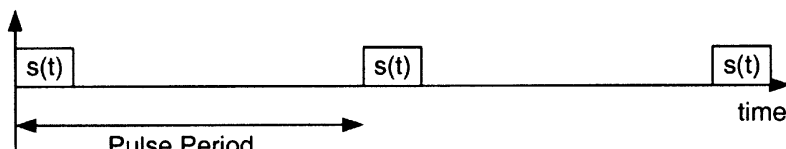

Pulse based Orthogonal Frequency Division Multiplexing (OFDM)

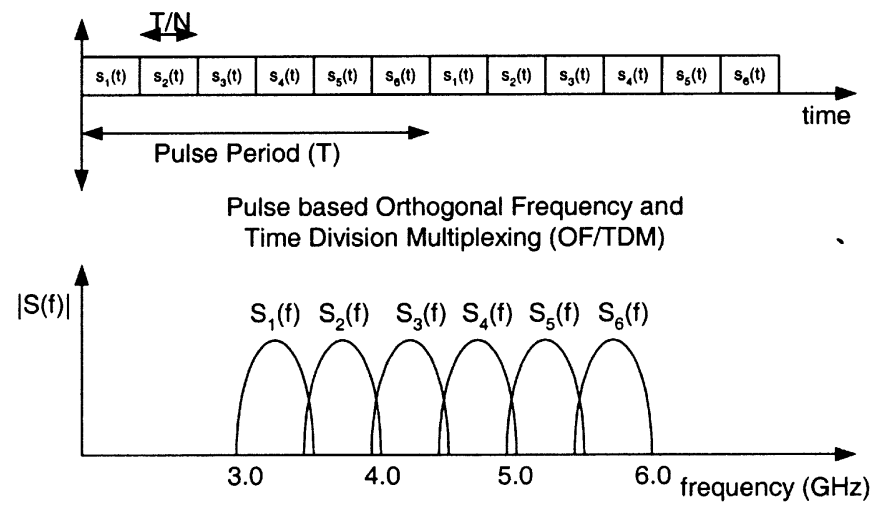

$s(t)=s_{1}(t)+s_{2}(t)+s_{3}(t)+s_{4}(t)+s_{5}(t)+s_{6}(t)$ : Concatenated OFDM waveform

$s_{i}(t)$ : Sub-band waveform

$\mathrm{N}$ : Number of sub-bands $(\mathrm{N}=6$ in this example)

Fig. 9. Pulsed orthogonal multiband system.

Although UWB is intended for supporting high data rate communications, in practice it must network devices over a range of data rates depending on device capabilities and types of applications, e.g., low-rate data connection between a mouse/keyboard and desktop PC to very high rate streaming of digital audio/video between a source (DVD player, etc.) and a high-resolution (flat panel plasma) display. The range of applications indicate that the MAC protocol functionalities must include support for both delay sensitive isochronous flows that require QoS guarantees as well delay tolerant random access. The MAC is expected to support some amount of limited portability/mobility that will lead to uncoordinated deployment where device clusters or piconets can be collocated or significantly overlapping and maintain aforementioned QoS over wireless links characterized by wireless links whose loss rates are sensitive to the (time-varying) interference environment. However, it is anticipated that each cluster will contain a coordinator ${ }^{12}$ which allows the possibility of MAC layer scheduling (i.e., controlled resource allocation) that is known to be consistent with providing good QoS performance. However, near-far issues due to collocated piconets is expected to arise in some scenarios that will need resolution via adoption of appropriate multiple access/scheduling approaches in conjunction with link layer strategies to manage interference. These indicate the need to consider mechanisms for interference avoidance via orthogonal spectral shaping designs and/or receiver approaches for MAI mitigation such as multiuser detection.

\footnotetext{
${ }^{12}$ In some scenarios, the choice of the coordinator is obvious-such as the host PC communicating with its client devices. In others that consist mainly of peer-to-peer devices, a mechanism to elect a coordinator will be needed.
}

\section{Multiband UWB Systems}

The considerations mentioned in the earlier sections may motivate a shift in UWB system design away from a traditional impulse-based radio that uses all the $3-10 \mathrm{GHz}$ band simultaneously in favor of a multibanded design where transmissions are staggered in time across the constituent bands. Consider a pulsed multiband system where one of several (typically 3-10) subbands are used sequentially for transmission. This helps limit the dynamic range of the received signal—such band hopping is reminiscent of traditional narrowband frequency-hopped systems. One of the main advantages of a multiband system is the ability for more fine-grained control of the transmit PSD so as to maximize the average transmit power while meeting the spectral mask. Further, narrower subband bandwidths ease the requirement on analog-to-digital converter (ADC) sampling rates (vis-à-vis a full-band receiver) and, consequently, facilitates greater digital processing. Nonetheless, the bandwidth in each subband is wide enough to allow a combination of options inclusive of direct sequence spreading, orthogonal frequency division multiplexing (OFDM) and coded (im)pulse modulation as shown in Fig. 9. This allows for tradeoffs between the time-domain pulsing and frequency-domain spreading in order to obtain desired performance in multipath channels and in the presence of interference from other UWB users in an uncoordinated network setting. Another ensuing advantage to the receiver design is that a lower complexity RAKE per subband (as compared to a RAKE receiver that spans the entire 3-10 $\mathrm{GHz}$ band) suffices for energy capture and mitigating the MAI.

In multiband UWB designs, the subband bandwidth is bounded below by the FCC minimum bandwidth (500 $\mathrm{MHz}$ ) constraint. The optimal choice is governed by many 


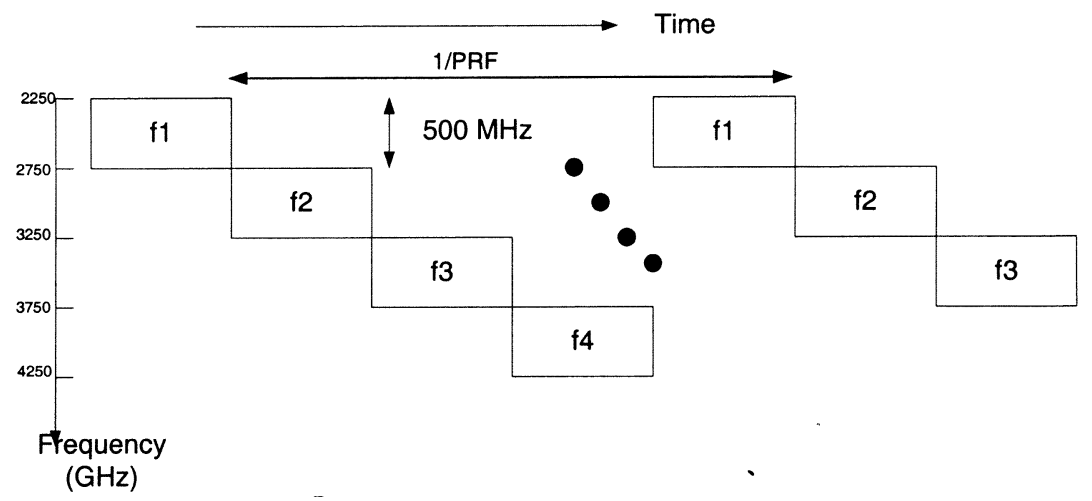

Fig. 10. Band-hopped multiband system.

considerations-the desired peak throughput rate, the in-band multipath channel, and MAI interference, as well as hardware constraints arising from practical considerations such as ADC sampling rates and cost. A possible receiver architecture consists of a single I-Q downconversion receiver (i.e., a direct conversion receiver) that switches with the band-hopping rate. The performance of such an architecture in multipath is fundamentally limited by the energy capture ability because when the receiver switches to demodulating the subsequent subband, it loses any ability to capture the multipath dispersed energy from the current subband. Thus, in order to improve the energy capture, pulsed multiband UWB systems need slower time-frequency hopping, i.e., longer contiguous symbol transmissions in each subband. This has naturally led to OFDM instead of pure pulse modulation in each subband due to the former's inherent robustness to multipath.

Multiple piconet coexistence is enabled in multiband UWB systems by introducing channelization via use of suitably designed frequency-hopping sequences over the set of subbands as in Fig. 10-in principle, depending on the channel environment and desired data rates, the hop rate can be slow (multiple symbols sent on one subband prior to band switching) or fast (only one symbol sent per subband). The frequency-hopping sequences are designed to minimize "collision" events when two users in different piconets simultaneously use the same subband-such cases lead to erasure of the transmitted symbols. Thus, the number of simultaneously operating piconets that can be supported by this approach depends on the availability of frequency-hopping sequences with one coincidence property [35] that are known to be optimal in suppressing multiuser interference (MUI) in AWGN. In the presence of multipath, MUI is exacerbated due to overlap of symbols transmitted on adjacent time-frequency slots by different users. Thus, additional robustness must be obtained through time-frequency diversity in the modulation process via suitable combination of coding across subbands and spreading in time. In situations where severe near-far MUI is a problem, multiband UWB systems may also have the option of fallback modes in which the piconets abandon the time-frequency hopping codes, and organize themselves into frequency division multiplexing usage of the overall spectrum-e.g., by each piconet picking one particular, unique subband to operate in. Conversely, in normal operating conditions, additional bands may be added to increase the aggregate data rate.

In summary, one of the more important attributes of such multiband schemes is that they provide necessary flexibility to balance the often conflicting choices while providing a pathway to match link parameters to prevailing channel conditions. Dynamic frequency selection (DFS) is, thus, an important attribute of future agile radios that must sense the interference environment and take appropriate action to avoid low quality subbands, e.g., the U-NII bands when a 802.11 interferer is operational. Such flexibility is particularly important given the nascent stage of global UWB regulations as well the variation in existing interference scenarios.

\section{A. Multipath Channel Modeling}

System design for any new technology must start with a fundamental knowledge of the propagation channel. Measurement studies are particularly challenging in this case due to the large bandwidths and frequency bands involved-reported studies over the 3-10 GHz range are few and far between. Intel Corp. has performed several channel measurements spanning the frequency spectrum from 2-8 GHz [12] that were submitted to the IEEE 802.15.3 subcommittee on this topic. Typical channel realizations from this study point to two important characteristics of a very wideband, indoor channel. First, the multipath spans several nanoseconds in time which results in intersymbol interference (ISI) if UWB pulses are closely spaced in time. Second, the very wide bandwidth of the transmitted pulse results allows fine resolution of multipath components. This is both good and bad-on one hand, fine resolution implies that each multipath component undergoes less amplitude fluctuations (fading) due to fewer interfering reflections and the potential for significant diversity gains due to the large number of available paths. On the other hand, the average total received energy is distributed over a (possibly large) number of paths; the implication of this on system design is discussed in the next section.

Based on the deliberations in the IEEE 802.15.3a channel modeling task group that considered various proposals that optimally chose the respective model parameters (e.g., mean excess and root mean square (RMS) delay, mean number of 
significant paths) to best fit the observed data, a slightly modified version of the Saleh-Valenzuela $(\mathrm{S}-\mathrm{V})$ model for indoor channels [15] was adopted. The channel measurements showed a clustering of the multipath arrivals which is best captured by the $\mathrm{S}-\mathrm{V}$ model. In addition, the amplitude statistics of the measurements was found to fit the log-normal or Nakagami distribution rather than the Rayleigh [11], [12]; thus, the original S-V model was accordingly modified.

The proposed multipath model for system performance evaluation consists of discrete time impulse response represented as

$$
h(t)=\sum_{k=0}^{L} \sum_{k=0}^{K} \alpha_{k, l} \delta\left(t-T_{l}-\tau_{k, l}\right)
$$

where $\alpha_{k, l}$ are the gain coefficients, $T_{l}$ is the delay of the $l$ th cluster, and $\tau_{k, l}$ is the delay of the $k$ th multipath component in the $l$ th cluster relative to the cluster arrival time. Two other parameters required to complete the specification are $\Lambda$, the cluster arrival rate, and $\lambda$, the ray arrival rate (arrival of paths within a cluster). Thus, the distribution of the cluster arrival time and the ray arrival time are given by

$$
\begin{aligned}
p\left(T_{l} \mid T_{l-1}\right) & =\Lambda \exp \left[-\Lambda\left(T_{l}-T_{l-1}\right], l>0\right. \\
p\left(\tau_{k, l} \mid \tau_{(k-1) l}\right) & =\lambda \exp \left[-\lambda\left(\tau_{k, l}-\tau_{(k-1) l}\right)\right], k>0 .
\end{aligned}
$$

A proposed model for the gains is $\alpha_{k, l}=p_{k, l} \beta_{k, l}$ where $p_{k, l}$ is equi-probable \pm 1 and $\beta_{k, l}$ is a log-normal random variable specified by $|\beta| \propto 10^{n / 20}$ where $n \sim \mathcal{N}\left(\mu_{k, l}, \sigma^{2}\right)$. The $\mu_{l}$ is related to model parameters via

$$
\mu_{l}=\frac{10 \ln \left(\Omega_{0}\right)-\frac{10 T_{l}}{\Gamma}-\frac{10 \tau_{k, l}}{\gamma}}{\ln (10)}-\frac{\sigma^{2} \ln (10)}{20}
$$

where $\sigma^{2}$ is obtained from the data set.

Table 2 provides a summary of results from model fitting for a set of representative channel scenarios (LOS and NLOS) based on extensive measurements conducted by Intel Corp. for typical indoor apartment dwellings. ${ }^{13}$

Table 2 shows that, for a 167 psec multipath resolution, corresponding to a bandwidth of $6 \mathrm{GHz}$, more than 30 significant paths may exist; this represents a significant challenge for UWB receivers as a large number of paths must be coherently combined for sufficient symbol energy. In addition, maximum excess delay spreads greater than 60-70 ns. (RMS delay of the order of 20-25 ns) were commonly observed, which suggests that some type of ISI mitigation might be required for very high rate implementations. This is verified by measured channel traces such as the one shown in Fig. 11. For the interested reader, the detailed channel model adopted by IEEE 802.15.3a task group is available at [13].

\section{B. Single-User Performance in Multipath}

As already emphasized, typical NLOS indoor environments give rise to dense multipath that will disperse the transmitted energy over a large number of component

\footnotetext{
${ }^{13}$ The total average received power of each realization is normalized to one in order to provide a fair comparison with other wideband and narrowband systems.
}

Table 2

Multipath Channel Characteristics and Model Parameters.

\begin{tabular}{c|c|c}
\hline Channel Characteristics & LOS & NLOS \\
\hline Mean Excess Delay $(\mathrm{ns}) \tau_{m}$ & 4 & 17 \\
\hline RMS Delay $(\mathrm{ns}) \tau_{r m s}$ & 9 & 15 \\
\hline NP (10 dB) & 7 & 35 \\
\hline Model Parameters & & \\
\hline$\Lambda(1 / \mathrm{ns})$ & $1 / 60$ & $1 / 11$ \\
\hline$\lambda(1 / \mathrm{ns})$ & $1 / 0.5$ & $1 / 0.35$ \\
\hline$\gamma$ & 1.6 & 8.5 \\
\hline$\sigma(\mathrm{dB})$ & 4.8 & 4.8 \\
\hline
\end{tabular}

paths. ${ }^{14}$ Hence for reliable detection, UWB receivers must capture a sufficient amount of the multipath energy by using a RAKE receiver with multiple arms. Since UWB is targeted at bit rates $>100 \mathrm{Mb} / \mathrm{s}$ (symbol duration of $<10 \mathrm{~ns}$ ), the delay spread could result in possibly significant ISI impacting several symbol durations that may require mitigation via (symbol-rate) equalization post-RAKE combining. With increasing desired bit rates, the cost/complexity of the RAKE and equalizer will become an important determinant in the cost/complexity tradeoffs in transceiver design.

For a preliminary discussion, consider a total of $L$ resolvable multipath components with uniform power-delay profile (i.e., the average power on each path is constant). Assume that all $L$ RAKE fingers are used in the combiner, termed the All Rake. The performance of any Rake architecture can be quantified in terms of the combining gain defined as the ratio of the output (postcombining) average SNR to the input per-branch average SNR. Of the possible diversity combining approaches, maximum ratio combining (MRC) where the signal on each diversity path is weighted in proportion to the branch SNR and combined is known to be optimal (in the maximum-likelihood sense) and provides the maximum gain; in the case of perfect estimation, this equals $L$. An alternate combining scheme is equal gain combining (EGC) where all paths are weighted equally - this provides inferior SNR gain vis-à-vis MRC, but has the advantage of not requiring knowledge of the branch SNR (and, hence, the channel gain).

In reality, the multipath power-delay profile is always nonuniform with an approximate exponential decay among paths in a cluster. Thus, some form of selection RAKE combiner (alternatively termed as generalized selection combining) wherein only a subset of the available paths (i.e., for example the strongest $M$ out of $L$ paths) [9] will be typically used, implying an inherent tradeoff (for any given delay profile) between the amount of energy capture and RAKE complexity. Further, since channel coefficients are estimated independently, the quality of channel estimation (as determined by the error covariance of any estimator, say, based on a preamble) is dependant on the branch SNR. Nonuniform delay profiles raise the important issue as to the number of paths to include in a selection RAKE combiner. The heuristic is as follows: the weaker paths

\footnotetext{
${ }^{14}$ Table 1 shows that for a 167 psec multipath resolution, corresponding to a bandwidth of $6 \mathrm{GHz}$, there could be more than 30 significant paths.
} 


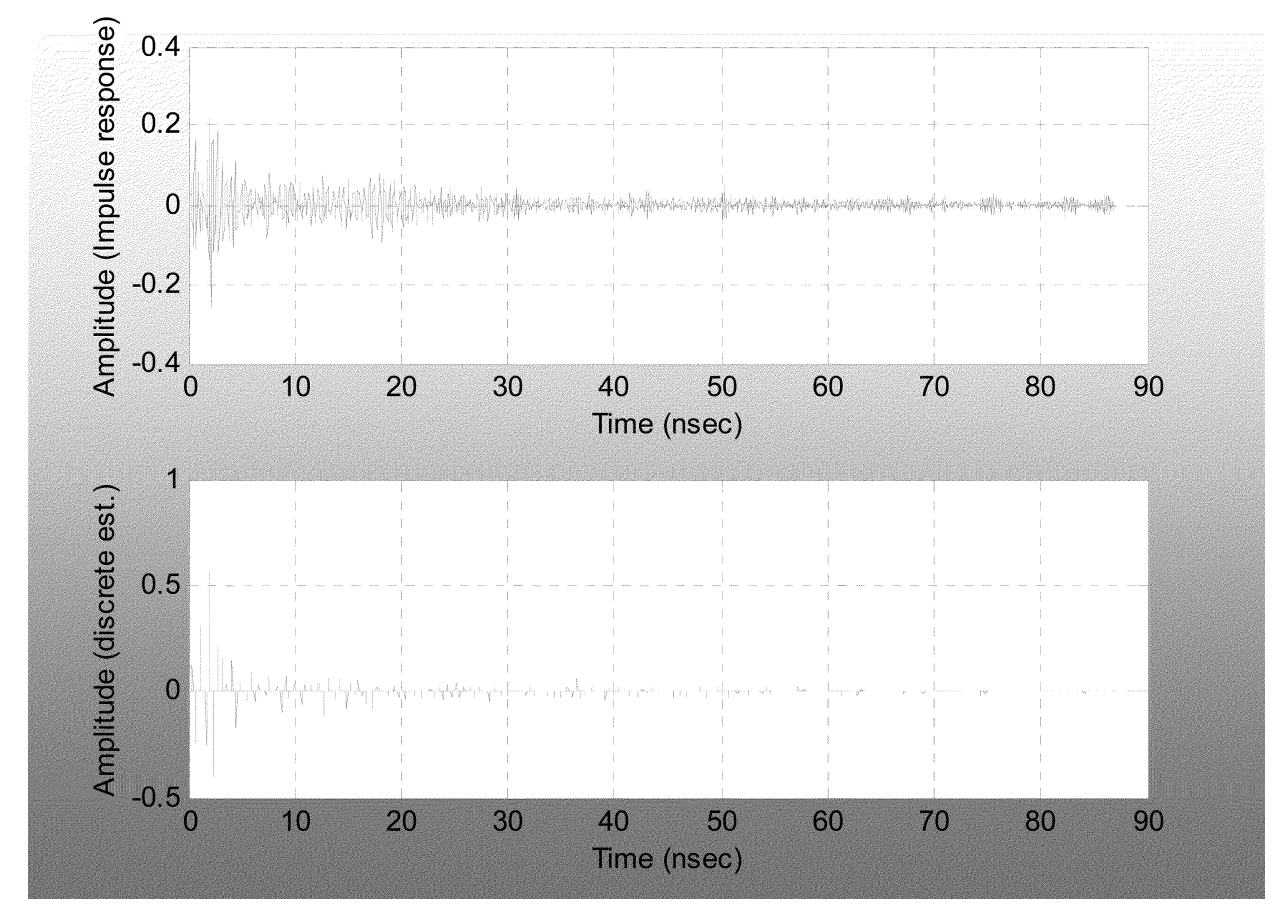

Fig. 11. UWB channel model realizations.

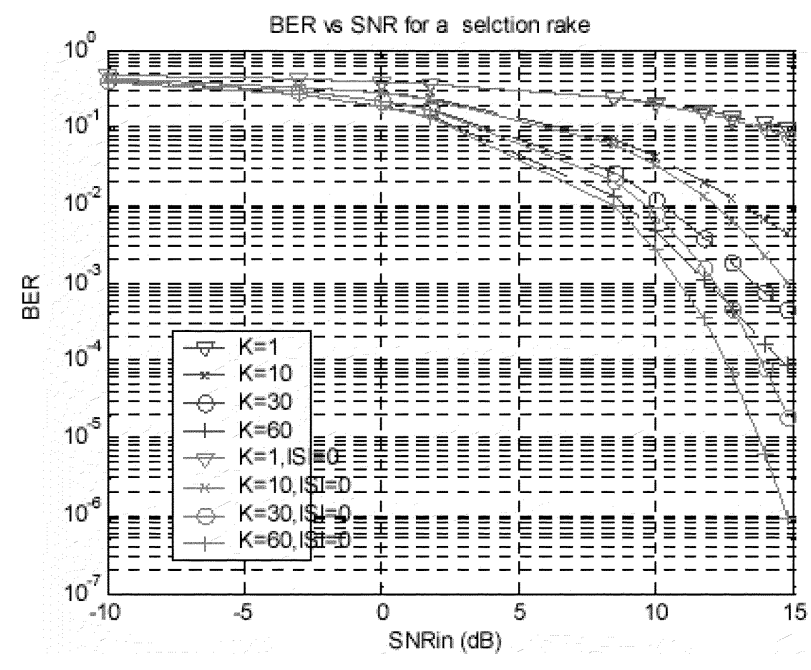

Fig. 12. UWB performance in multipath: selection diversity combining ( $K$ : number of paths combined).

contribute little energy to the combiner and, moreover, are most susceptible to estimation errors that degrade the output SNR; therefore, it is anticipated that an optimum number of paths exist (dependant on the specific delay profile) beyond which output SNR actually decreases. As a result, proper selection of the multipath components to be combined can help balance receiver complexity with desired performance. The above observations are supported by the simulation results shown in Figs. 12 and 13 using 10000 channel realizations based on the $\mathrm{S}-\mathrm{V}$ model with parameters in Table 2. MRC selection combining combined the strongest $K$ paths whereas the EGC combiner used the first $K$ arriving paths. In Fig. 12, the curves labeled "ISI=0" are

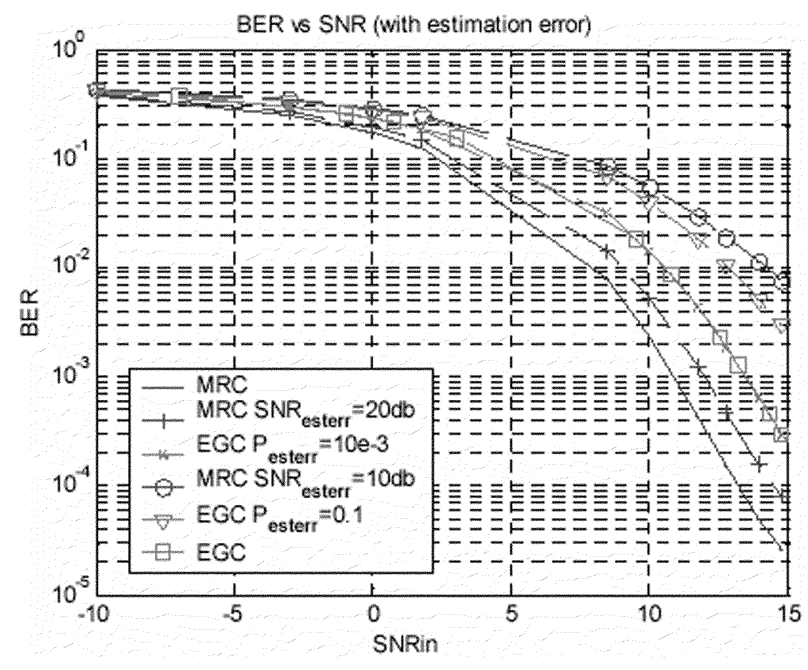

Fig. 13. Performance in multipath: sensitivity to channel estimation errors.

included as a baseline to indicate the amount of ISI energy collected by the RAKE as a function of increasing $K$; the close proximity of the ISI-free and RAKE performance curves indicate that for the S-V model, RAKE performance degradation due to ISI is relatively minor. Fig. 13 quantifies the impact of channel estimation error for both MRC and EGC; in the former, channel estimation error was simulated by adding an independent Gaussian noise term of variance $\sigma_{\text {err }}^{2}$. This variance is chosen relative to the strongest path amplitude $h_{\max }$ in each realization, i.e., the performance curves are labeled by $\mathrm{SNR}_{\text {esterr }}=h_{\max }^{2} / \sigma_{\mathrm{err}}^{2}$. In case of EGC with BPSK modulation, channel error is equivalent to an incorrect determination of the sign of any multipath 


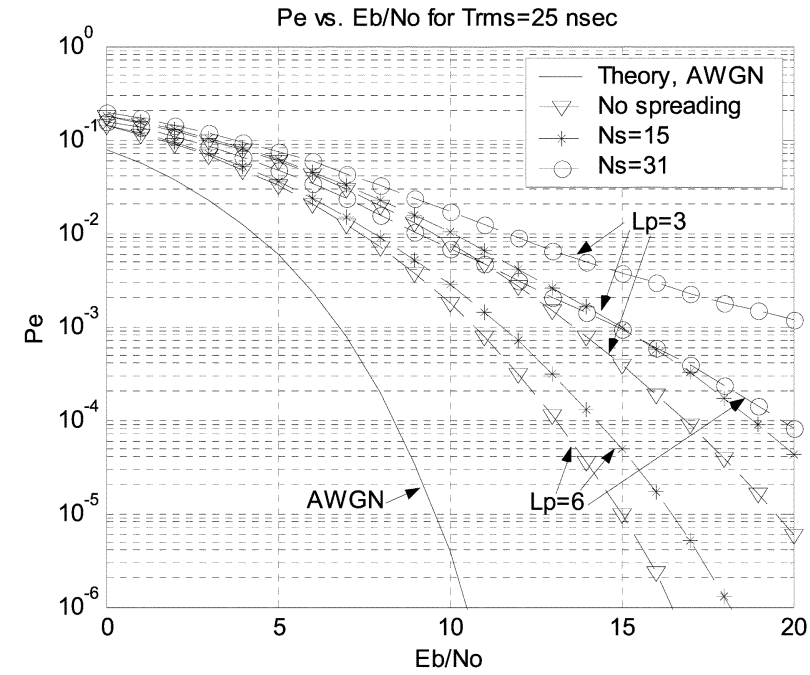

Fig. 14. Performance in multipath: pulse versus DS-UWB performance.

component; thus, EGC curves are labeled with a probability of error $P_{\text {esterr }}$. While both schemes degrade, EGC is more robust as expected.

Finally, Fig. 14 provides a comparison of the performance of a pulse UWB ("no spreading") system with DS-UWB in NLOS multipath channel with RMS delay spread $25 \mathrm{~ns}$ averaged over 50 realizations [29]. A 100-Mb/s system with binary modulation was used with the number of RAKE arms $L_{p}=3,6$. The results suggests that spreading using DS-UWB actually performs worse than a pulse-UWB system attributable to the increased interchip interference (due to proximity of the chip pulses in DS-UWB) and the nonideal autocorrelation of the spreading sequence chosen. This indicates the importance of code design to optimize DS-UWB performance in such dense multipath situations, and constitutes a significant design challenge for DS-UWB designs when scaling to higher rates (i.e., lower spreading sequence lengths).

\section{UWB Coexistence With NB Sources}

Adequate coexistence of UWB with WLAN systems such as $802.11 \mathrm{a}$ will be a necessity for deployment given that WLAN and high-rate WPAN systems are likely to be located in close proximity in various devices such as desktop PCs and, more critically, on mobile devices such as laptops (where the separation between UWB and WLAN transceiver may be only a few inches). The necessary coexistence solutions will include both physical and MAC layer components, but in this paperk we focus only on link layer approaches.

There are many factors which affect how UWB impacts existing "narrowband" systems-notably the separation between the devices, the channel propagation losses, the modulation technique, the PRF employed by the UWB system, and the receiver antenna gain of the "narrowband" receiver in the direction of the UWB transmitter. In other words, while a detailed analysis of this important issue is beyond the scope of this work, useful preliminary insight is nonetheless obtained by modeling the interference caused by a UWB transmitter as a wideband white Gaussian source that has the effect of raising the noise floor of the "narrowband" receiver-as will be shown, such AWGN approximation for pulse UWB in some cases performs adequately. A UWB receiver is also potentially susceptible to traditional narrowband transmitters that operate within its passband. However, 802.11a WLAN transmitters will appear as strong narrowband interferers (typical SIR $=-40 \mathrm{~dB}$ or more) to UWB receiver in a known band, and can be adequately excised with adaptive filtering techniques that have been well-documented in the literature for narrowband overlay of DS-SS systems [20], [21]. Accordingly, we ignore the issue of narrowband interference rejection at the UWB transceiver and focus on the impact of UWB source on a proximate NB receiver.

Approximate Analysis Based on AWGN Approximation for $U W B$ Source: The received waveform at the input to the NB receiver is assumed to be given by

$$
r(t)=s(t)+u(t)+n(t)
$$

where $u(t)$ is the UWB interferer, $n(t)$ is standard complex baseband AWGN with PSD $N_{0}$, and the desired NB signal is given by

$$
s(t)=\sqrt{2 P_{s}} \cos \left(\omega_{0} t+\theta\right) \sum_{k} b_{k} v\left(t-k T_{s}\right)
$$

assuming BPSK modulation with average transmit power $P_{s}$ at a carrier frequency $f_{0}$ with random phase $\theta$, with symbol time $T_{s}$. Further, the transmitted narrowband waveform power is normalized to one, i.e., $1 / T_{s} \int v^{2}(t) d t=1$. The UWB interference is modeled simply as a binary $( \pm 1)$ modulated pulse train with average power $P_{u}$, i.e.,

$$
u(t)=\sqrt{P_{u}} \sum_{i} a_{i} w\left(t-i T_{r}\right)
$$

where $T_{r}$ is the pulse repetition period, and the transmitted pulse waveform is power normalized, i.e., $1 / T_{r} \int|w(t)|^{2} d t=1$. Note that further specific details of the UWB modulation are not relevant, since we assume that the UWB waveform is spectrally flat with PSD given by the FCC Part 15 limits over a bandwidth $B_{u} \gg B_{r}=1 / T_{r}$.

The narrowband receiver is assumed to employ a coherent matched filter to the received waveform whose output is sampled at the optimal time and input to a binary slicer for the final bit decision. Invoking the AWGN approximation for the UWB source allows us to apply well-known results for the BER for BPSK modulation with a few simple modifications, as explained next. This requires the introduction of a key parameter $N_{p}=B_{r} / B_{s}^{n b}$, which is recognized as the ratio of the PRF to the NB signal bandwidth (also the BW of the NB receiver).

1) For $N_{p} \gg 1$, a large number of UWB pulses are contained within a symbol time of the NB waveform, and it is anticipated that the central limiting arguments provide adequate justification for the AWGN assumption. In this case, the BER of the NB system is given by

$$
P_{e}=\frac{1}{2} \operatorname{erfc}\left(\gamma_{a}\right)
$$




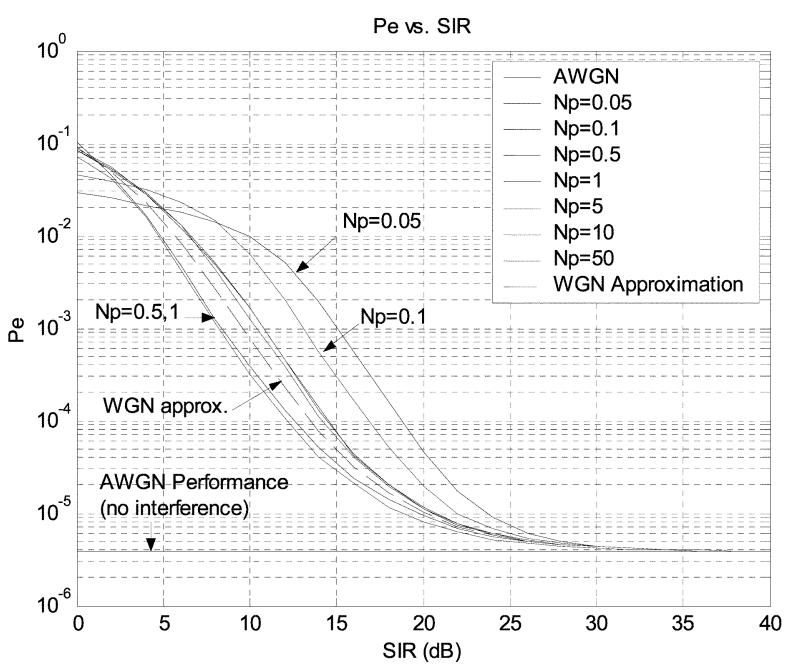

Fig. 15. Coexistence: performance of BPSK in UWB

where $\gamma_{a}=\sqrt{1 /\left(\left(1 / E_{s} / N_{0}\right)+1 / L_{i}\right)}$ where $E_{s}=$ $P_{s} T_{s}$ is the average bit energy of the NB signal and $L_{i}=P_{s} /\left(P_{\mathrm{usd}} B_{s}\right)$ with $P_{\mathrm{usd}}$ denoting the Part 15 PSD. Thus, $L_{i}$ denotes the signal-to-interference ratio within the bandwidth of the NB receiver; note that in the absence of the UWB interferer, the expression reverts to the familiar BPSK error rate.

2) When $N_{p}<1$, some NB system bits are impacted by a UWB pulse while others are not. Thus, considering a large number of transmitted bits, $N_{p}\left(1-N_{p}\right)$ represents the long-term fraction of time that a UWB interferer is present (absent), hence

$$
P_{e}=N_{p} \frac{1}{2} \operatorname{erfc}\left(\gamma_{a}\right)+\left(1-N_{p}\right) \frac{1}{2} \operatorname{erfc}\left(\sqrt{\frac{E_{s}}{N_{0}}}\right)
$$

where $\gamma_{a}$ is defined as above.

Results from computation using the above approximation as well as Monte Carlo simulation using 10000 realizations are shown in Figs. 15 and 16. The narrowband system is assumed to be ISI free, with root-raised cosine filters at both the transmitter and receiver, with a rolloff factor of 0.25 ; the performance of the narrowband receiver is plotted as a function of the SIR for SNR $=E_{b} / N_{0}=10 \mathrm{~dB}$, and bipolar PAM modulated UWB interference. These results show that there is significantly greater potential for interference when the PRF is low (i.e., low $N_{p}$ ) relative to the bandwidth of the narrowband receiver. Also, the simulation results can be up to $5 \mathrm{~dB}$ worse than that based on modeling the interference as WGN. The plot also shows that for high PRFs (relative to the bandwidth of the narrowband receiver), there is only about a 1-dB difference between the actual (simulated) performance and the WGN approximation as could be intuitively expected.

The impact of modulation choice of the narrowband system is investigated in Fig. 16, which considers a binary pulse position modulation system (2-PPM) in place of BPSK-UWB as was assumed in Fig. 15. It is of interest to note that the BER performance worsens significantly as the PRF increases, in direct contrast with the BPSK result-this is attributable to the presence of spikes in the transmit PSD

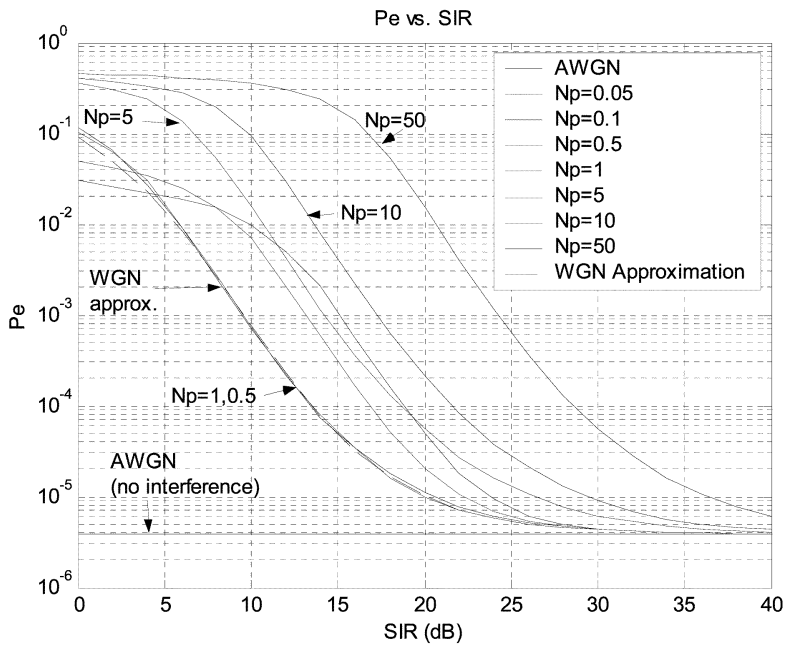

Fig. 16. Coexistence: performance of binary PPM in UWB.

that increase with PRF. It has been demonstrated that the impact of UWB interference to PPM systems could be reduced by increasing the level of randomness in pulse positioning, such as by enlarging the step size of data modulation in TH-PPM.

\section{Timing Acquisition}

Timing acquisition (along with frequency and channel estimation) is typically performed using a preamble in packet data systems; ${ }^{15}$ thus, in high data rate applications, preamble efficiency is desired so to minimize the throughput loss. To see this, consider a 1024-B data payload transmitted at $100 \mathrm{Mb} / \mathrm{s}$; a $10-\mu \mathrm{s}$ preamble overhead amounts to an overhead of $11 \%$ in this case, which rises to $34 \%$ for $500-\mathrm{Mb} / \mathrm{s}$ data rate.

For acquisition of a single user's preamble sequence in AWGN, a matched filter or correlator receiver is optimal. However, implementing a digital filter matched to the UWB pulse is infeasible, as it would require excess of gigahertz sampling rates that currently exceeds the state of the art of reasonable ADC designs in terms of cost and power consumption. Thus, a running analog correlator is employed for timing acquisition as shown in Fig. 15. The step size of a serial search algorithm for determining the optimum pulse timing is dependant on the main lobe width of the autocorrelation function of the UWB pulse, which is typically on the order of $0.1 \mathrm{~ns}$. Hence, while fast timing acquisition remains an important goal of any such packet-based system architecture, the near impulse like (ideal) autocorrelation property of the UWB pulse presents a significant obstacle in achieving this. To illustrate this consider a $100-\mathrm{Mb} / \mathrm{s}$ system, for which the range of the search equals one pulse repetition period (= $10 \mathrm{~ns}$ ); since the autocorrelation of a typical UWB pulse has an effective duration of approximately $0.1 \mathrm{~ns}$, the serial correlator step size for timing adjustment must be no greater than $0.1 \mathrm{~ns}$. To satisfy a receiver operating curve (ROC) point

\footnotetext{
${ }^{15}$ It suffices to think of the preamble as a suitably chosen binary \pm 1 modulated sequence of UWB pulses. Thus, the acquisition operation can be conceptualized as obtaining the pulse timing followed by phase alignment of the preamble sequence.
} 
characterized respectively by false alarm and missed detection probability of $P_{\mathrm{fa}}=P_{\mathrm{md}}=10^{-5}$ for a detector input $E_{b} / N_{0}=5 \mathrm{~dB}$ per pulse, it was determined that a preamble sequence length of $N_{\mathrm{pr}}=11$ would be required. Combining this information leads to an estimate of the time it takes to search once through the entire range as

$$
T_{\text {search }}=\frac{10}{0.1} \times N_{\mathrm{pr}} \times 10 \mathrm{~ns}=11 \mu \mathrm{s} .
$$

Note that the above represents a worst case estimate of the acquisition time ignoring occurrences of false alarms (that may occasionally extend the acquisition times beyond the above) in AWGN. In the presence of multipath, the above simple estimate needs to be suitably amended by considering the dispersal of the transmitted energy among the components. We assume that in this case, timing acquisition is obtained based on a single - the strongest-path component. By considering the probability density function of the amplitudes over a large number of channel realizations obtained from the Intel study, it was found that the strongest path contained $>10 \%$ of the total power more than $95 \%$ of the time. As a heuristic, we estimate the impact of this energy reduction per pulse on the search time by dividing the value obtained earlier by 0.1 (the energy reduction factor), yielding $110 \mu \mathrm{s}$. This indicates the difficult choices confronting the system architect in this regard; in principle, a general strategy to reduce the search time to an acceptable value (say 11 $\mu \mathrm{s}$ as in the AWGN case) would require multiple correlators running in parallel, at additional hardware expense. For example, dividing the search space into ten (equal) disjoint bins and assigning one correlator to search each bin sequentially would reduce the acquisition time to order of $11 \mu \mathrm{s}$, which could be further reduced possibly by optimizing the search strategy (e.g., random search) across the bins based on knowledge of the power delay profile.

\section{E. Additional Systems Architecture and Circuit Implementation Issues}

Other important architectural challenges include efficient circuitry for UWB pulse generation, broadband antenna design and power-efficient transceivers constitute other major circuit implementation challenges. UWB pulses have typical durations of hundreds of picoseconds and, as has already been exemplified, proper pulse shape design is key to meeting the Part 15 spectral masks. Controlling the response of very broadband transmit and receive antennas is a considerable challenge; accordingly, the received pulse at the receive input after the RF stage which is the convolution of the generated baseband pulse shape (UWB transmitter output) and the antenna responses is not precisely known, even in the absence of multipath. Consequently, coherent reception requiring a correlation becomes a practical difficulty even in an AWGN environment, since the correlation receiver needs to accurately reproduce the equivalent transmitted pulse shape seen at the receiver. Differential modulation schemes that obviate this have been considered in [36] and [37] — this allows the receiver to correlate using the received signal itself that is an imperfect replica of the true transmitted UWB pulse shape. While this leads to a mismatched correlation (i.e., implying some performance penalty vis-à-vis coherent correlator), the design has some attractive features including the potential for a low-cost implementation as well as the ability to provide easier RAKE combining of multipath energy.

Since many UWB applications are expected to support user mobility, the net transceiver power budget is an important consideration. A key advantage of UWB designs is that highly linear power amplifiers are not required because the UWB pulse generator need only produce a peak-to-peak voltage swing on the order of $100 \mathrm{mV}$ to meet the FCC spectral mask requirements that can be achieved by a suitable UWB waveform choice. While the low power of UWB emissions indicates the potential of greater integration of baseband and RF circuits into CMOS, ${ }^{16}$ there exist some significant challenges. A notable one is the specification (number of bits and sampling clock rate) of the ADCs needed in any UWB transceiver; architectures that require sampling of the UWB signal at the pulse rate (typically a few $\mathrm{GHz}$ ) with $8-10 \mathrm{~b}$ of resolution are infeasible for integration into a volume product with present-day state of the art due to the high cost and power consumption that would be incurred.

In this context, a multiband architecture where the transmission across subbands are staggered in time provide potential solutions to some of the key problems noted above with a (single-band) ultrawideband receiver. First, this permits the use of relatively narrower band RF front ends that allow digitization of a subband signal using sampling rates that are lower by a factor equal to the number of subbands as compared to a single ultrawideband channel. This allows efficient receiver design with current $\mathrm{ADCs}$ with fewer bits of resolution (2-4 b/sample) that suffices for lower order modulation. Nonetheless, like all design approaches, the multiband has its own challenges. Prominent among them is the need for a fast frequency-hopping generator (typical switching rate of 100 $\mathrm{MHz}$ ) circuit that is low power and has good transient characteristics. Further, while fewer bits/sample is consistent with cost-effective implementation, this would potentially impact AGC operation due to reduced ability in identifying and rejecting strong in-band interferers as a result of the insufficient resolution.

\section{Conclusion AND Future Challenges}

This paper has provided a tutorial overview of system design aspects of UWB radios highlighting both the potential for high-rate, short-range wireless communications as well as the implementation and design challenges that must be confronted. A low-cost, low-power design that meets the varied application scenario calls for a scalable and flexible system architecture that provides good overlay capability and is robust to multipath fading. We identified some key link layer subsystems for which innovative design solutions are needed, namely: 1) timing acquisition; 2) energy capture in

\footnotetext{
${ }^{16}$ Since voltage levels available in CMOS can be reduced in order to provide faster speeds according to Moore's Law, low peak powers are desirable to support a fully integrated RF front end in CMOS with no external power amplifier.
} 
multipath; and 3) mechanisms for coexistence with existing narrowband users in an overlay scenario.

Several aspects of UWB for short-range wireless networking have not been touched upon in this article and remain areas of considerable interest. Primary among this is the potential for multihop networking using such short-range (UWB) connections as may be necessary for some in-home distribution applications. In such cases, it is not clear from a cost/complexity tradeoff whether such longer range single-hop networks would be preferred (as in 802.11 LAN) to a multihop UWB network and remains an important avenue for further investigations.

\section{REFERENCES}

[1] "FCC notice of proposed rule making, revision of part 15 of the commission's rules regarding ultra-wideband transmission systems," Federal Communications Commission, Washington, DC, ET-Docket 98-153.

[2] J. D. Taylor, Introduction to Ultra Wideband Radar Systems. Boca Raton, FL: CRC, 1995.

[3] R. Fontana. A brief history of UWB communications. [Online]. Available: http://www.multispectral.com

[4] J. McCorkle. (2000) A tutorial on ultrawideband technology. [Online]. Available: www.ewh.ieee.org/r3/central_virginia/ html/pastmeetings/uwb-oct03.pdf

[5] R. A. Scholtz, "Multiple access with time-hopping impulse modulation," presented at the Conf. Milcom, Bedford, MA, 1993.

[6] M. Z. Win and R. A. Scholtz, "Ultra-wide bandwidth time hopping spread-spectrum impulse radio for wireless multiple access communications," IEEE Trans. Commun., vol. 48, pp. 679-690, Apr. 2000.

[7] — "Impulse radio: How it works," IEEE Commun. Lett., vol. 2, pp. 36-38, Feb. 1998.

[8] - "On the robustness of ultra-wide bandwidth signals in dense multipath environments," IEEE Commun. Lett., vol. 2, pp. 51-53, Feb. 1998.

[9] — " "On the energy capture of ultrawideband signals in dense multipath environments," IEEE Commun. Lett., pp. 245-247, Sept. 1998.

[10] D. Cassioli, M. Win, F. Vatalaro, and A. Molisch, "Performance of low complexity rake reception in a realistic UWB channel," in Proc. ICC2002, pp. 763-767.

[11] D. Cassioli, M. Z. Win, and A. F. Molisch, "The ultra widebandwidth indoor channel: From statistical model to simulations," IEEE J. Select. Areas Commun., pp. 1247-1257, Aug. 2002.

[12] "UWB Channel Modeling Contribution," Intel Corp., IEEE P802.15-02/02 790r0-SG3a.

[13] "Channel Modeling Sub-Committee Final Report," IEEE, IEEE P802.15-02/02 490r0-SG3a.

[14] "SG3a Alternate PHY Selection Criteria," IEEE, IEEE P802.15-02/105r25, 2002.

[15] A. A. Saleh and R. Valenzuela, "A statistical model for indoor multipath propagation," IEEE J. Select. Areas Commun., vol. 5, pp. 128-137, Feb. 1987.

[16] G. Durisi and G. Romano, "On the validity of the Gaussian approximation to characterize the multiuser capacity of UWB TH-PPM," in Proc. IEEE Conf. UWB Systems and Technologies, 2002, pp. 255-258.

[17] S. Verdu, "Spectral efficiency in the wideband regime," IEEE Trans. Inform. Theory, vol. 48, pp. 1319-1343, June 2002.

[18] I. E. Telatar and D. N. C. Tse, "Capacity and mutual information of wideband multipath fading channels," IEEE Trans. Inform. Theory, vol. 46, pp. 1384-1400, July 2000.

[19] M. Medard and R. Gallager, "Bandwidth scaling for fading multipath channels," IEEE Trans. Inform. Theory, vol. 48, pp. 840-852, Apr. 2002.

[20] L. B. Milstein, "Wideband code division multiple access," IEEE J. Select. Areas Commun., pp. 1344-1354, Aug. 2000.

[21] J. Wang and L. B. Milstein, "CDMA overlay situations for microcellular mobile communications," IEEE Trans. Commun., vol. 43, pp. 603-614, Feb.-Apr. 1995.

[22] J. R. Foerster, E. Green, V. S. Somayazulu, and D. Leeper, "Ultra-wide band technology for short or medium-range wireless communications," Intel Tech. J., vol. Q2, 2001.
[23] D. Leeper, "A long term view of short range wireless," IEEE Computer, vol. 34, pp. 39-44, June 2001.

[24] F. Ramirez-Mireles, "On performance of ultra-wideband signals in Gaussian noise and dense multipath," IEEE Trans. Veh. Technol., vol. 50, pp. 244-249, Jan. 2001.

[25] _ - "Performance of ultra wideband SSMA using time hopping and M-ary PPM," IEEE J. Select. Areas Commun., vol. 19, pp. 1186-1196, June 2001.

[26] V. S. Somayazulu, "Multiple access performance in UWB systems using time hopping vs. direct sequence spreading," presented at the IEEE Wireless Communications and Networking Conf., Orlando, FL, 2001.

[27] M. Welborn, "System considerations for ultra-wideband wireless networks," presented at the IEEE Radio and Wireless Conf., Bedford, MA, 2001.

[28] M. Welborn et al., "Multi-user perspectives in UWB communiction networks," presented at the 2002 IEEE Conf. UWB, Baltimore, MD, 2002.

[29] J. R. Foerster, "The effects of multipath interference on the performance of UWB systems in an indoor wireless channel," presented at the IEEE Vehicular Technology Conf., Rhodes, Greece, 2001.

[30] _ - "The performance of a direct-sequence spread ultra-wideband system in the presence of multipath, narrowband interference and multiuser interference," presented at the IEEE UWB Workshop, Baltimore, MD, 2002.

[31] J. D. Choi and W. Stark, "Performance of UWB communications with suboptimal receivers in multipath channels," IEEE J. Select. Areas Commun., vol. 20, pp. 1754-1766, Dec. 2002.

[32] J. Proakis, Digital Communications, 2nd ed. New York: McGrawHill, 1994.

[33] "Ultrawideband radio in multiaccess wireless communications," IEEE J. Select. Areas Commun. (special issue), vol. 20, pp. 1733-1744, Dec. 2002.

[34] IEEE Conf. Ultra Wideband Systems and Technologies.

[35] P. Fan and M. Darnell, Sequence Design for Communication Applications. New York: Wiley, 2000.

[36] R. T. Hoctor and H. W. Tomlinson, "An overview of delay-hopped, transmitted reference RF communications," General Electric, R\&D Tech. Rep. 2001CRD198, 2002.

[37] M. Ho, V. S. Somayazulu, J. Foerster, and S. Roy, "A differential detector for an ultra-wideband communications systems," presented at the Spring VTC, Huntsville, AL, 2002.

[38] M. Hamalainen, V. Hovinen, and J. Iinatti, "Performance comparison between various UWB signals in AWGN channel in presence of multitone interference at the GSM downlink," in Proc. 4th Int. Symp. Wireless Personal Multimedia Communications, 2001, pp. 449-453.

[39] A. Swami, B. Sadler, and J. Turner, "On the coexistence of ultrawideband and narrowband radio systems," presented at the 1st IEEE UWB Workshop, Baltimore, MD, 2002.

[40] V. S. Somayazulu, J. Foerster, and S. Roy, "Design challenges for very high data rate UWB systems," presented at the Proc. Asilomar Conference, Pacific Grove, CA, 2002.

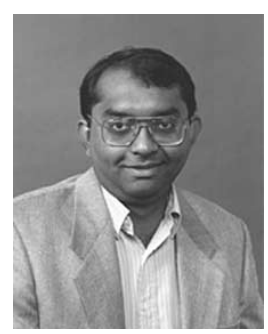

Sumit Roy received the B.Tech. degree from the Indian Institute of Technology, Kanpur, in 1983, the M.S. and Ph.D. degrees in electrical engineering from the University of California, Santa Barbara, in 1985 and 1988 respectively, and the M.A. degree in statistics and applied probability from the University of California, Santa Barbara, in 1988.

He was with the Moore School of Electrical Engineering, University of Pennsylvania, and the University of Texas, San Antonio. He is currently Professor of Electrical Engineering, University of Washington, Seattle. From 2001 to 2003, he was with Intel Wireless Technology Lab, Hillsboro, $\mathrm{OR}$, as a Senior Researcher engaged in development of ultrawideband systems technology for high-speed wireless access. He currently serves on the Editorial Board for the Journal of Wireless Communications and Mobile Computing. His research interests include analysis/design of communication systems/networks, with an emphasis on next-generation mobile and wireless networks.

Dr. Roy's activities for the IEEE Communications Society include membership of several technical and conference program committees. He recently completed a term as Editor for the IEEE TRANSACTIONS ON WIRELESS COMMUNICATIONS. 


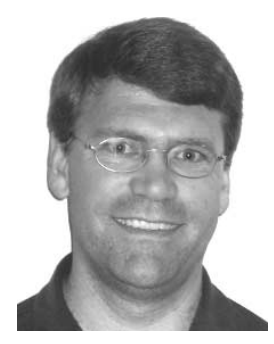

Jeff R. Foerster received the B.S., M.S., and $\mathrm{Ph} . \mathrm{D}$. degrees from the University of California, San Diego.

He worked on Broadband Wireless Access (BWA) systems and standards (IEEE 802.16). $\mathrm{He}$ is currently a Wireless Researcher with Intel Wireless Technology Lab, Hillsboro, OR. His graduate school research focused on adaptive interference suppression and coding techniques for CDMA systems. He is currently focusing on future short- and medium-range wireless technologies, including ultrawideband (UWB) technology and related regulations, system design, and performance analysis.

Dr. Foerster chaired the channel modeling subcommittee for the IEEE 802.15.SG3a study group focusing on UWB channel models to be used for evaluating future UWB-based proposals.

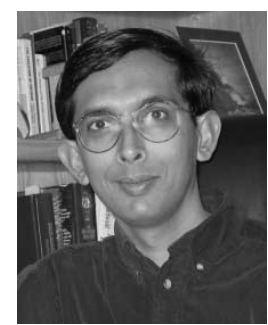

V. S. Somayazulu received the B.Tech. degree from the Indian Institute of Technology, Madras, and the M.S. and Ph.D. degrees from the University of California, Santa Barbara.

$\mathrm{He}$ is a Senior Staff Researcher with the Radio Communication Lab within Intel R\&D, Hillsboro, OR, which has a broad charter to conduct research to enable Intel's leadership in the wireless communications area. He has worked, among other areas, on communications system design for digital TV broadcast systems and IS-95 and W-CDMA based cellular systems as well as OFDM based WLAN systems. His current interests lie in the areas of UWB and OFDM system design at the physical layer, and MAC issues for high speed multimedia wireless networks.

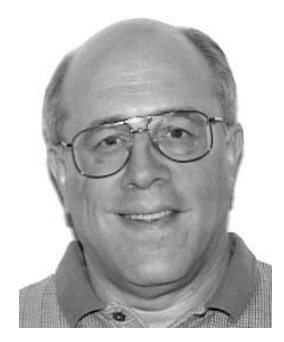

Dave G. Leeper received the B.S. degree in electrical engineering from Washington University, St. Louis, MO, in 1969, the M.S. degree in electrical engineering from Cornell University, Ithaca, NY, in 1970, and the Ph.D. degree in electrical engineering from the University of Pennsylvania, Philadelphia, in 1977.

He began his career at AT\&T Bell Labs and has held officer-level positions at Bellcore and Motorola. He has 35 years of experience in digital communications, software, system architecture, wireless technology, engineering, and management. He is currently a Principal Engineer in Intel's Analog and Radio Operation, Intel Wireless Technology Lab, Hillsboro, OR. He has eight patents granted or pending and has published papers in Scientific American, various IEEE journals, and the Intel Technology Journal. His favorite activity is working with small entrepreneurial teams on new technologies and business concepts. 